\title{
Electrophilic Fluorodesilylation of Allenylmethylsilanes: a Novel Entry to 2-Fluoro-1,3-dienes
}

\section{$M^{\mathrm{a}}$ Carmen Pacheco and Véronique Gouverneur}

\author{
University of Oxford, Chemistry Research Laboratory, Mansfield Road, OX1 3TA, \\ Oxford (UK)
}

\section{SUPPORTING INFORMATION}

General. Infrared spectra were recorded on a Paragon 1000 FT-IR spectrometer and only peaks of interest are reported. ${ }^{1} \mathrm{H}$ NMR were recorded on Bruker DPX 200, DPX 400 and AV 400 spectrometers, at a frequency of 200 and $400 \mathrm{MHz}$ respectively. Chemical shifts are reported in ppm and coupling constant $(J)$ are given in hertz $(\mathrm{Hz})$. ${ }^{13} \mathrm{C}$ NMR were recorded on a Bruker DPX 200, AV 400 and AMX500 spectrometers at a frequency of 50,100 or $125 \mathrm{MHz}$ respectively with $\mathrm{CDCl}_{3}$ or $\mathrm{CD}_{3} \mathrm{COCD}_{3}$ as the internal reference. Mass spectra $(\mathrm{m} / \mathrm{z})$ were recorded on Micromass GCT in Chemical Ionisation $\left(\mathrm{NH}_{3}, \mathrm{CI}^{+}\right)$or Electron Impact (EI). Analytical thin layer chromatography (TLC) was performed on Merck Silica gel $60 \mathrm{~F}_{254}$ plates. All reactions were carried out under an argon atmosphere in dried glassware with magnetic stirring. Compounds $\mathbf{1 f}-\mathbf{i}$, $\mathbf{2 f - i}$ and 3a-i have been previously reported.

Preparation of alkynols 3. These alkynols were prepared following 6 protocols: A, B, C, D, E, and F.

Protocol A: To a stirred solution of aldehyde $(4 \mathrm{mmol})$ in dry THF $(5.2 \mathrm{~mL})$ was added a solution of 1-propynylmagnesium bromide $(8.8 \mathrm{~mL}, 4.4 \mathrm{mmol}, 0.5 \mathrm{M})$ in THF at $0{ }^{\circ} \mathrm{C}$ under an argon atmosphere. The resulting solution was allowed to warm up to room temperature and stirred for $6 \mathrm{~h}$. The reaction mixture was then cooled to $0{ }^{\circ} \mathrm{C}$ and an aqueous saturated solution of $\mathrm{NH}_{4} \mathrm{Cl}(10 \mathrm{~mL})$ was added. The mixture was extracted with $\mathrm{Et}_{2} \mathrm{O}(3 \times 12 \mathrm{~mL})$ and washed with brine $(2 \times 7 \mathrm{~mL})$. The organic layer was dried over $\mathrm{MgSO}_{4}$, followed by removal of the solvent at reduced pressure. The resulting crude product was purified by silica gel column chromatography.

Protocol B: ${ }^{1}$ A solution of $n$-butyllithium in hexane $(2.21 \mathrm{~mL}, 3.53 \mathrm{mmol}, 1.6$ M) was added to a solution of the corresponding acetylene $(3.53 \mathrm{mmol})$ in dry THF at $78^{\circ} \mathrm{C}$ under an argon atmosphere. After 30 min stirring at this temperature, the aldehyde (2.94 mmol) was added. The reaction mixture was allowed to warm up to $0{ }^{\circ} \mathrm{C}$, and stirring was continued at this temperature for $2 \mathrm{~h}$. The reaction was quenched with $\mathrm{H}_{2} \mathrm{O}$ $(10 \mathrm{~mL})$ and extracted with $\mathrm{Et}_{2} \mathrm{O}(3 \times 12 \mathrm{~mL})$. The organic phase was dried over $\mathrm{MgSO}_{4}$ and the solvent concentrated at reduced pressure. The resulting crude product was purified by silica gel column chromatography.

Protocol C: ${ }^{2}$ To a solution of silylated alkynol $(5.588 \mathrm{mmol})$ in THF $(3 \mathrm{~mL})$ was added a solution of $n$-Bu $\mathrm{Bu}_{4}(8.73 \mathrm{~mL}, 8.731 \mathrm{mmol}, 1.0 \mathrm{M})$ in THF. After the reaction mixture was stirred at room temperature for $2.5 \mathrm{~h}$, brine was added and the mixture was extracted with $\mathrm{Et}_{2} \mathrm{O}(3 \times 15 \mathrm{~mL})$. The combined organic phases were dried over $\mathrm{MgSO}_{4}$ and the solvent concentrated at reduced pressure. The resulting crude product was purified by silica gel column chromatography.

\footnotetext{
${ }^{1}$ Ajamian, A.; Gleason, J. L. Org. Lett. 2003, 5, 2409-2411.

${ }^{2}$ Matsuda, F.; Kawatsura, M.; Hosaka, K.-i.; Shirahama, H. Chem. Eur. J. 1999, 5, 3252-3259.
} 
Protocol D: ${ }^{3}$ Acetic anhydride $(189 \mu \mathrm{L}, 2 \mathrm{mmol})$ was added to a solution of 2butyn-1,4-diol (344 mg, $4 \mathrm{mmol})$ in pyridine $(4 \mathrm{~mL})$, and the mixture was stirred at $0{ }^{\circ} \mathrm{C}$ for $3 \mathrm{~h}$. The solvent was then evaporated at reduced pressure. The resulting crude product was purified by silica gel column chromatography.

Protocol E: ${ }^{4}$ To a suspension of sodium hydride $(60 \%$ dispersion in mineral oil, $0.680 \mathrm{~g}, 17 \mathrm{mmol})$, previously washed with hexane $(2 \times 10 \mathrm{~mL})$, in DMF $(20 \mathrm{~mL})$ was added 2-butyne-1,4-diol (1.722 g, $20 \mathrm{mmol})$. The reaction mixture was stirred at room temperature for $3 \mathrm{~h}$, and benzyl bromide $(2.022 \mathrm{~mL}, 17 \mathrm{mmol})$ was added via syringe. The mixture was stirred for $20 \mathrm{~h}$, quenched with $\mathrm{H}_{2} \mathrm{O}(50 \mathrm{~mL})$, and the aqueous layer was extracted with $\mathrm{Et}_{2} \mathrm{O}(4 \times 50 \mathrm{~mL})$. The combined organic layers were dried over $\mathrm{MgSO}_{4}$ and the solvent concentrated at reduced pressure. The resulting crude product was purified by silica gel column chromatography.

Protocol F: ${ }^{5}$ A suspension of iodobenzene $(560 \mu \mathrm{L}, 5 \mathrm{mmol})$, propargyl alcohol (291 $\mu \mathrm{L}, 5 \mathrm{mmol}$ ), dichlorobis(triphenylphosphine)palladium(II) (35 mg, $0.05 \mathrm{mmol}$ ), copper(I) iodide $(1.4 \mathrm{mg}, 0.025 \mathrm{mmol})$, and diethylamine $(8 \mathrm{~mL})$ was stirred under an argon atmosphere at room temperature. After $21.5 \mathrm{~h}$, the diethylamine was removed, and aqueous $\mathrm{HCl}(0.5 \mathrm{M}, 20 \mathrm{~mL})$ was added. The reaction mixture was extracted with $\mathrm{Et}_{2} \mathrm{O}(3 \times 20 \mathrm{~mL})$ and washed with $\mathrm{H}_{2} \mathrm{O}(2 \times 15 \mathrm{~mL})$. The organic phase was dried over $\mathrm{MgSO}_{4}$ and the solvent concentrated at reduced pressure. The resulting crude product was purified by silica gel column chromatography.

$\begin{array}{ll}\mathrm{PhH}_{2} \mathrm{CH}_{2} \mathrm{C} & \mathrm{CH}_{3} \begin{array}{l}\text { 1-Phenyl-4-hexyn-3-ol (3a): }{ }^{6} \text { Protocol A was followed; }{ }^{1} \mathrm{H} \\ 2 \mathrm{H}), 2.79(\mathrm{t}, 2 \mathrm{H}, J=8.0 \mathrm{~Hz}), 4.33-4.37(\mathrm{~m}, 1 \mathrm{H}), 7.18-7.23\end{array}\end{array}$ (m, 3H), 7.26-7.32 (m, 2H); ${ }^{13} \mathrm{C}$ NMR (100 MHz, $\left.\mathrm{CDCl}_{3}\right) \delta$ 3.5, 31.4, 39.5, 62.0, 80.1, 81.4, 125.9, 128.36, 128.44, 141.4; IR $\left(\mathrm{v}, \mathrm{cm}^{-1}\right) 3357(\mathrm{OH}), 2233(\mathrm{C} \equiv \mathrm{C}), 1603,1496$ (Ar); $\mathrm{MS}\left(\mathrm{GCT}, \mathrm{CI}^{+}\right): \mathrm{m} / z 192\left(\mathrm{MNH}_{4}^{+}\right)$.

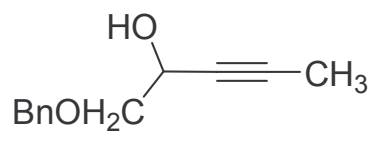

1-(Benzyloxy)-3-pentyn-2-ol (3b): ${ }^{7}$ Protocol A was followed; ${ }^{1} \mathrm{H}$ NMR $\left(200 \mathrm{MHz}, \mathrm{CDCl}_{3}\right) \delta 1.84(\mathrm{~d}, 3 \mathrm{H}, J=2.2 \mathrm{~Hz}), 2.01$ (bs, 1H), 3.47-3.65 (m, 2H), 4.49-4.58 (m, 1H), $4.61(\mathrm{~d}, 2 \mathrm{H}, J=$ $1.4 \mathrm{~Hz}), 7.32-7.38(\mathrm{~m}, 5 \mathrm{H}) ;{ }^{13} \mathrm{C} \mathrm{NMR}\left(100 \mathrm{MHz}, \mathrm{CDCl}_{3}\right) \delta 3.4,61.5,73.1,73.7,76.9$, 81.7, 127.6, 128.2, 137.5; IR ( $\left.v, \mathrm{~cm}^{-1}\right) 3423(\mathrm{OH}), 2241(\mathrm{C} \equiv \mathrm{C}), 1605,1497$ (Ar), 1110 (C-O); $\mathrm{MS}\left(\mathrm{GCT}, \mathrm{CI}^{+}\right): \mathrm{m} / z 208\left(\mathrm{MNH}_{4}{ }^{+}\right)$.

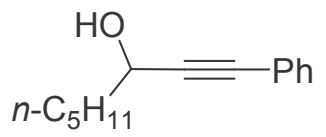

1-Phenyl-1-octyn-3-ol (3c): ${ }^{8}$ Protocol B was followed; ${ }^{1} \mathrm{H}$ NMR $\left(400 \mathrm{MHz}, \mathrm{CDCl}_{3}\right) \delta 0.91(\mathrm{t}, 3 \mathrm{H}, J=7.2 \mathrm{~Hz}), 1.32-1.36(\mathrm{~m}, 4 \mathrm{H})$, $1.50-1.56(\mathrm{~m}, 2 \mathrm{H}), 1.77-1.83(\mathrm{~m}, 2 \mathrm{H}), 2.14(\mathrm{~s}, 1 \mathrm{H}), 4.58-4.62(\mathrm{~m}$, $1 \mathrm{H}), 7.30-7.31(\mathrm{~m}, 3 \mathrm{H}), 7.42-7.45(\mathrm{~m}, 2 \mathrm{H}) ;{ }^{13} \mathrm{C} \mathrm{NMR}\left(100 \mathrm{MHz}, \mathrm{CDCl}_{3}\right) \delta 14.0,22.5$, 24.9, 31.4, 37.8, 62.9, 84.7, 90.2, 122.6, 128.2, 128.3, 131.6; IR (v, $\left.\mathrm{cm}^{-1}\right) 3332(\mathrm{OH})$, 1599, 1490 (Ar); MS (GCT, $\left.\mathrm{CI}^{+}\right): m / z 203\left(\mathrm{MH}^{+}\right)$.

\footnotetext{
${ }^{3}$ Osa, Y.; Kobayashi, S.; Sato, Y.; Suzuki, Y.; Takino, K.; Takeuchi, T.; Miyata, Y.; Sakaguchi, M.; Takayanagi, H. J. Med. Chem. 2003, 46, 1948-1956.

${ }^{4}$ Wipf, P.; Rahman, L. T.; Rector, S. R. J. Org. Chem. 1998, 63, 7132-7133.

${ }^{5}$ Wrobel, J.; Li, Z.; Dietrich, A.; McCaleb, M.; Mihan, B.; Sredy, J.; Sullivan, D. J. Med. Chem. 1998, 41, 1084-1091.

${ }^{6}$ Harada, T.; Katsuhira, T.; Osada, A.; Iwazaki, K.; Maejima, K.; Oku, A. J. Am. Chem. Soc. 1996, 118, 11377-11390.

${ }^{7}$ Marshall, J. A.; Wang, X.-j. J. Org. Chem. 1996, 56, 960-969.

${ }^{8}$ Ahn, J. H.; Joung, M. J.; Yoon, N. M. J. Org. Chem. 1995, 60, 6173-6175.
} 


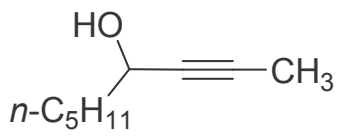

2-Nonyn-4-ol (3d): ${ }^{9}$ Protocol A was followed; ${ }^{1} \mathrm{H}$ NMR $(200$ $\left.\mathrm{MHz}, \mathrm{CDCl}_{3}\right) \delta 0.89(\mathrm{t}, 3 \mathrm{H}, J=6.5 \mathrm{~Hz}), 1.24-1.48(\mathrm{~m}, 6 \mathrm{H}), 1.58-$ $1.69(\mathrm{~m}, 2 \mathrm{H}), 1.81(\mathrm{~d}, 3 \mathrm{H}, J=2.2 \mathrm{~Hz}), 2.10(\mathrm{~s}, 1 \mathrm{H}), 4.25-4.32(\mathrm{~m}$, $1 \mathrm{H}) ;{ }^{13} \mathrm{C}$ NMR $\left(50 \mathrm{MHz}, \mathrm{CDCl}_{3}\right) \delta 3.4,13.9,22.5,24.8,31.4,38.0,62.6,80.5,80.7$; IR $\left(v, \mathrm{~cm}^{-1}\right) 3357(\mathrm{OH}), 2234(\mathrm{C} \equiv \mathrm{C}) ; \mathrm{MS}\left(\mathrm{GCT}, \mathrm{CI}^{+}\right): \mathrm{m} / z 158\left(\mathrm{MNH}_{4}{ }^{+}\right)$.

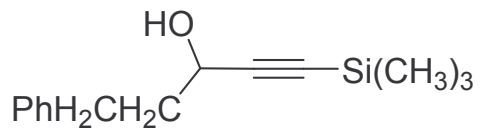

5-Phenyl-1-(trimethylsilyl)-1-pentyn-3-ol

(3e): ${ }^{10}$

Protocol B was followed; ${ }^{1} \mathrm{H}$ NMR $\left(400 \mathrm{MHz}, \mathrm{CDCl}_{3}\right) \delta$ $0.19(\mathrm{~s}, 9 \mathrm{H}), 1.78(\mathrm{bs}, 1 \mathrm{H}), 1.99-2.07(\mathrm{~m}, 2 \mathrm{H}), 2.80(\mathrm{t}$, $2 \mathrm{H}, J=7.8 \mathrm{~Hz}), 4.36(\mathrm{t}, 1 \mathrm{H}, J=6.6 \mathrm{~Hz}), 7.18-7.22(\mathrm{~m}, 3 \mathrm{H}), 7.27-7.31(\mathrm{~m}, 2 \mathrm{H}) ;{ }^{13} \mathrm{C}$ NMR $\left(100 \mathrm{MHz}, \mathrm{CDCl}_{3}\right) \delta-0.1,31.4,39.2,62.2,89.9,106.4,126.0,128.4,128.5$, 141.3; IR ( $\left.v, \mathrm{~cm}^{-1}\right) 3384(\mathrm{OH}), 2172(\mathrm{C} \equiv \mathrm{C}), 1604,1496$ (Ar), 844 (C-Si); MS (GCT, $\left.\mathrm{CI}^{+}\right): m / z 259\left(\mathrm{MNH}_{4}^{+}\right)$.

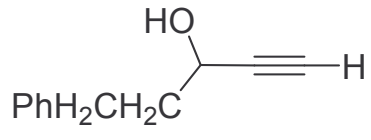

5-Phenyl-1-pentyn-3-ol (3f): ${ }^{2}$ Protocol $\mathrm{C}$ was followed; ${ }^{1} \mathrm{H}$ NMR (400 MHz, $\left.\mathrm{CDCl}_{3}\right) \delta 1.69(\mathrm{bs}, 1 \mathrm{H}), 2.00-2.09(\mathrm{~m}, 2 \mathrm{H})$, $2.52(\mathrm{~d}, 1 \mathrm{H}, J=2.0 \mathrm{~Hz}), 2.81(\mathrm{t}, 2 \mathrm{H}, J=7.8 \mathrm{~Hz}), 4.38(\mathrm{dt}, 1 \mathrm{H}, J$ $=6.6,2.0 \mathrm{~Hz}), 7.18-7.31(\mathrm{~m}, 5 \mathrm{H}) ;{ }^{13} \mathrm{C} \mathrm{NMR}\left(100 \mathrm{MHz}, \mathrm{CDCl}_{3}\right) \delta 31.2,39.1,61.6,73.4$,

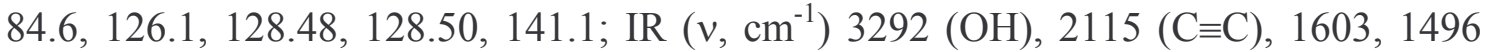
(Ar); $\mathrm{MS}\left(\mathrm{GCT}, \mathrm{CI}^{+}\right): m / z 178\left(\mathrm{MNH}_{4}{ }^{+}\right)$.

$\mathrm{HO}$

4-Hydroxybut-2-ynyl acetate (3g): ${ }^{3}$ Protocol D was followed; ${ }^{1} \mathrm{H}$ NMR $\left(400 \mathrm{MHz}, \mathrm{CDCl}_{3}\right) \delta 2.08(\mathrm{~s}, 3 \mathrm{H}), 3.29(\mathrm{~s}, 1 \mathrm{H}), 4.29(\mathrm{t}$, $2 \mathrm{H}, J=1.8 \mathrm{~Hz}), 4.69(\mathrm{~s}, 2 \mathrm{H}, J=1.8 \mathrm{~Hz}) ;{ }^{13} \mathrm{C} \mathrm{NMR}\left(100 \mathrm{MHz}, \mathrm{CDCl}_{3}\right) \delta 20.7,50.7$, 52.3, 79.4, 85.1, 170.4; IR ( $\left.\mathrm{v}, \mathrm{cm}^{-1}\right) 3418(\mathrm{OH}), 1745(\mathrm{C}=\mathrm{O}), 1231(\mathrm{C}-\mathrm{O})$; MS (GCT, $\left.\mathrm{CI}^{+}\right): m / z 146\left(\mathrm{MNH}_{4}^{+}\right)$.

$\mathrm{HO}$

4-(Benzyloxy)-2-butyn-1-ol (3h): ${ }^{4}$ Protocol E was followed; ${ }^{1} \mathrm{H}$ NMR $\left(400 \mathrm{MHz}, \mathrm{CDCl}_{3}\right) \delta 2.00(\mathrm{bs}, 1 \mathrm{H}), 4.22(\mathrm{t}, 2 \mathrm{H}, J=1.8$ $\mathrm{Hz}), 4.31(\mathrm{t}, 2 \mathrm{H}, J=1.8 \mathrm{~Hz}), 4.60(\mathrm{~s}, 2 \mathrm{H}), 7.28-7.36(\mathrm{~m}, 5 \mathrm{H}) ;{ }^{13} \mathrm{C}$ NMR $(100 \mathrm{MHz}$, $\left.\mathrm{CDCl}_{3}\right) \delta 50.8,57.3,71.6,81.3,84.9,127.9,128.0,128.4,137.2 ; \mathrm{IR}\left(\mathrm{v}, \mathrm{cm}^{-1}\right) 3385$ $(\mathrm{OH}), 1496(\mathrm{Ph}) ; \mathrm{MS}\left(\mathrm{GCT}, \mathrm{CI}^{+}\right): m / z 194\left(\mathrm{MNH}_{4}{ }^{+}\right)$.

$\mathrm{HO}$ 3-Phenyl-2-propyn-1-ol (3i): ${ }^{5}$ Protocol $\mathrm{F}$ was followed; ${ }^{1} \mathrm{H}$ NMR $\left(400 \mathrm{MHz}, \mathrm{CDCl}_{3}\right) \delta 1.69(\mathrm{~s}, 1 \mathrm{H}), 4.50(\mathrm{~s}, 2 \mathrm{H}), 7.30-7.33(\mathrm{~m}, 3 \mathrm{H})$, 7.43-7.45 (m, 2H); ${ }^{13} \mathrm{C}$ NMR $\left(100 \mathrm{MHz}, \mathrm{CDCl}_{3}\right) \delta 51.4,85.5,87.2,122.4,128.2,128.4$, 131.6; IR $\left(v, \mathrm{~cm}^{-1}\right) 3356(\mathrm{OH}), 2238(\mathrm{C} \equiv \mathrm{C}), 1599,1490(\mathrm{Ph}) ; \mathrm{MS}\left(\mathrm{GCT}, \mathrm{CI}^{+}\right): \mathrm{m} / \mathrm{z} 150$ $\left(\mathrm{MNH}_{4}^{+}\right)$.

\footnotetext{
${ }^{9}$ Midland, M. M.; Kazubski, A. J. Org. Chem. 1982, 47, 2814-2816.

${ }^{10}$ Bach, J.; Berenguer, R.; Garcia, J.; Loscertales, T.; Vilarrasa, J. J. Org. Chem. 1996, 61, 9021-9025.

${ }^{2}$ Matsuda, F.; Kawatsura, M.; Hosaka, K.-i.; Shirahama, H. Chem. Eur. J. 1999, 5, 3252-3259.

${ }^{3}$ Osa, Y.; Kobayashi, S.; Sato, Y.; Suzuki, Y.; Takino, K.; Takeuchi, T.; Miyata, Y.; Sakaguchi, M.; Takayanagi, H. J. Med. Chem. 2003, 46, 1948-1956.

${ }^{4}$ Wipf, P.; Rahman, L. T.; Rector, S. R. J. Org. Chem. 1998, 63, 7132-7133.

${ }^{5}$ Wrobel, J.; Li, Z.; Dietrich, A.; McCaleb, M.; Mihan, B.; Sredy, J.; Sullivan, D. J. Med. Chem. 1998, 41, 1084-1091.
} 
Preparation of mesylates 2. Triethylamine $(1.68 \mathrm{~mL}, 12.06 \mathrm{mmol})$ was added to a solution of alkynol $(6.03 \mathrm{mmol})$ in dry, alcohol free, $\mathrm{CH}_{2} \mathrm{Cl}_{2}(6 \mathrm{~mL})$ at $0{ }^{\circ} \mathrm{C}$, followed by addition of methanesulfonyl chloride $(701 \mu \mathrm{L}, 9.05 \mathrm{mmol})$. The reaction was allowed to warm up to room temperature and stir at this temperature for $3.5 \mathrm{~h} . \mathrm{H}_{2} \mathrm{O}$ $(15 \mathrm{~mL})$ was then added and the reaction mixture was extracted with $\mathrm{CH}_{2} \mathrm{Cl}_{2}(3 \times 15$ $\mathrm{mL}$ ), dried over $\mathrm{MgSO}_{4}$ and the solvent concentrated at reduced pressure.

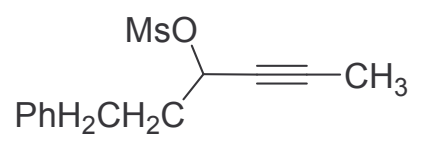

1-Phenyl-4-hexyn-3-ylmethanesulfonate (2a): ${ }^{1} \mathrm{H} \quad \mathrm{NMR}$ $\left(400 \mathrm{MHz}, \mathrm{CDCl}_{3}\right) \delta 1.91(\mathrm{~d}, 3 \mathrm{H}, J=2.4 \mathrm{~Hz}), 2.11-2.25(\mathrm{~m}$, $2 \mathrm{H}), 2.78-2.84(\mathrm{~m}, 2 \mathrm{H}), 3.11(\mathrm{~s}, 3 \mathrm{H}), 5.12-5.16(\mathrm{~m}, 1 \mathrm{H})$, 7.19-7.22 (m, 3H), 7.28-7.32 (m, 2H); ${ }^{13} \mathrm{C} \mathrm{NMR} \mathrm{(50} \mathrm{MHz,} \mathrm{acetone-d} 6$ ) $\delta 4.3,32.6,39.5$, 40.1, 73.2, 76.8, 87.4, 127.9, 130.2, 130.3, 142.2; IR ( $\left.\mathrm{cm}^{-1}\right) 2246(\mathrm{C} \equiv \mathrm{C}), 1604,1497$

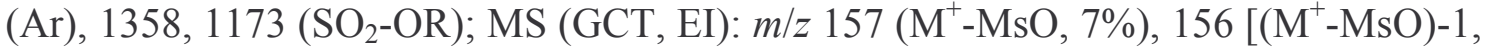
$100]$.<smiles>CC#CC(CO)OC</smiles>

1-(Benzyloxy)pent-3-yn-2-ylmethanesulfonate (2b): ${ }^{1} \mathrm{H}$ NMR $\left(200 \mathrm{MHz}\right.$, acetone- $\left.\mathrm{d}_{6}\right) \delta 1.88(\mathrm{~d}, 3 \mathrm{H}, J=2.2 \mathrm{~Hz}), 3.14(\mathrm{~s}, 3 \mathrm{H})$, $3.73(\mathrm{~d}, 2 \mathrm{H}, J=5.6 \mathrm{~Hz}), 4.63(\mathrm{~s}, 2 \mathrm{H}), 5.30-5.38(\mathrm{~m}, 1 \mathrm{H}), 7.27-$ $7.41(\mathrm{~m}, 5 \mathrm{H}) ;{ }^{13} \mathrm{C}$ NMR $\left(100 \mathrm{MHz}\right.$, acetone- $\left.\mathrm{d}_{6}\right) \delta 4.3,40.1,73.0,73.5,74.6,87.72$, 87.75, 129.46, 129.52, 130.1, 139.8; IR ( $\left.v, \mathrm{~cm}^{-1}\right) 2247(\mathrm{C} \equiv \mathrm{C}), 1605,1497$ (Ar), 1360, 1176 (SO $\left.{ }_{2}-\mathrm{OR}\right)$; MS (GCT, EI): $m / z 267\left(\mathrm{M}^{+}-1,2 \%\right), 172$ [( $\left.\left.\mathrm{M}^{+}-1\right)-\mathrm{MsO}, 100\right]$.

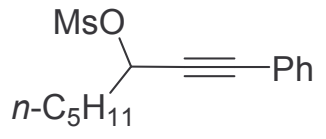

1-Phenyl-1-octyn-3-ylmethanesulfonate (2c): ${ }^{1} \mathrm{H}$ NMR $(400$ $\mathrm{MHz}$, acetone- $\left.\mathrm{d}_{6}\right) \delta 0.91(\mathrm{t}, 3 \mathrm{H}, J=6.4 \mathrm{~Hz}), 1.35-1.40(\mathrm{~m}, 4 \mathrm{H})$, 1.54-1.63 (m, 2H), 1.95-2.00 (m, 2H), $3.24(\mathrm{~s}, 3 \mathrm{H}), 5.44(\mathrm{t}, 1 \mathrm{H}, J=$

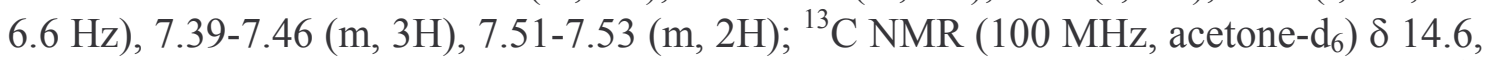
23.5, 25.6, 32.2, 37.0, 39.5, 73.1, 86.3, 89.0, 122.9, 129.9, 130.6, 133.0; IR (v, $\left.\mathrm{cm}^{-1}\right)$ $2232(\mathrm{C} \equiv \mathrm{C}), 1604,1491$ (Ar), 1363, $1176\left(\mathrm{SO}_{2}-\mathrm{OR}\right)$; MS (GCT, EI): m/z $185\left(\mathrm{M}^{+}-\right.$ $\mathrm{MsO}, 10 \%), 184\left[\left(\mathrm{M}^{+}-\mathrm{MsO}\right)-1,100\right]$.<smiles>CC#CC(OC)c1ccccc1</smiles>

2-Nonyn-4-ylmethanesulfonate (2d): ${ }^{1} \mathrm{H}$ NMR $(400 \mathrm{MHz}$, $\left.\mathrm{CDCl}_{3}\right) \delta 0.89(\mathrm{t}, 3 \mathrm{H}, J=6.8 \mathrm{~Hz}), 1.30-1.33(\mathrm{~m}, 4 \mathrm{H}), 1.46-1.49$ $(\mathrm{m}, 2 \mathrm{H}), 1.79-1.90(\mathrm{~m}, 5 \mathrm{H}), 3.10(\mathrm{~s}, 3 \mathrm{H}), 5.11-5.15(\mathrm{~m}, 1 \mathrm{H}) ;{ }^{13} \mathrm{C}$ $\operatorname{NMR}\left(50 \mathrm{MHz}, \mathrm{CDCl}_{3}\right) \delta 3.5,13.9,22.3,24.4,31.0,35.9,39.0,72.7,75.1,85.4$; IR (v, $\left.\mathrm{cm}^{-1}\right) 2244(\mathrm{C} \equiv \mathrm{C}), 1360,1176\left(\mathrm{SO}_{2}-\mathrm{OR}\right)$; MS (GCT, EI): m/z $123\left(\mathrm{M}^{+}-\mathrm{MsO}, 60 \%\right), 122$ $\left[\left(\mathrm{M}^{+}-\mathrm{MsO}\right)-1,100\right]$.

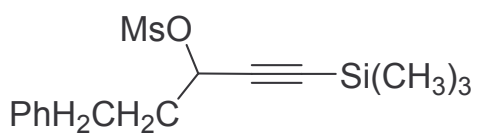

5-Pheny-1-(trimethylsilyl)-l-pent-1-yn-3ylmethanesulfonate (2e): ${ }^{1} \mathrm{H}$ NMR $\left(400 \mathrm{MHz}, \mathrm{CDCl}_{3}\right) \delta$ $0.20(\mathrm{~s}, 9 \mathrm{H}), 2.12-2.28(\mathrm{~m}, 2 \mathrm{H}), 2.79-2.85(\mathrm{~m}, 2 \mathrm{H}), 3.13$ $(\mathrm{s}, 3 \mathrm{H}), 5.13(\mathrm{t}, 1 \mathrm{H}, J=6.0 \mathrm{~Hz}), 7.19-7.23(\mathrm{~m}, 3 \mathrm{H}), 7.28-7.32(\mathrm{~m}, 2 \mathrm{H}) ;{ }^{13} \mathrm{C} \mathrm{NMR}(100$ $\mathrm{MHz}, \mathrm{CDCl}_{3}$ ) -0.4, 30.9, 37.2, 39.2, 71.6, 94.9, 100.1, 126.3, 128.5, 128.6, 140.0; IR ( $\left.\mathrm{cm}^{-1}\right)$ 1604, 1497 (Ar), 1362, 1175 (SO $\left.\mathrm{S}_{2}-\mathrm{OR}\right), 847$ (C-Si); MS (GCT, EI): m/z $215\left(\mathrm{M}^{+}-\right.$ $\mathrm{MsO}, 5 \%), 214\left[\left(\mathrm{M}^{+}-\mathrm{MsO}\right)-1,100\right]$.

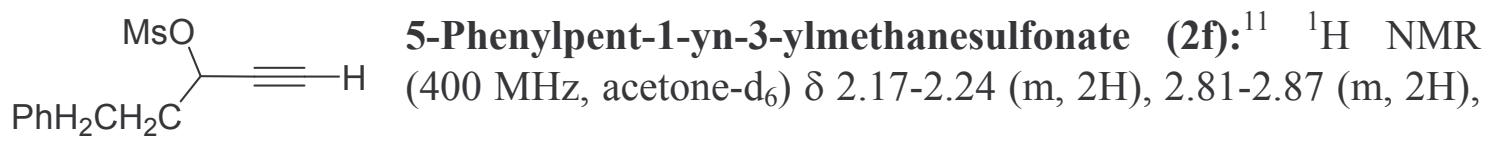

${ }^{11}$ Katsuhira, T.; Harada, T.; Maejima, K.; Osada, A.; Oku, A. J. Org. Chem. 1993, 58, 6166-6168. 
$3.20(\mathrm{~s}, 3 \mathrm{H}), 3.46(\mathrm{~d}, 1 \mathrm{H}, J=2.0 \mathrm{~Hz}), 5.22(\mathrm{dt}, 1 \mathrm{H}, J=6.6,2.0 \mathrm{~Hz}), 7.19-7.33(\mathrm{~m}, 5 \mathrm{H})$; ${ }^{13} \mathrm{C}$ NMR $\left(125 \mathrm{MHz}\right.$, acetone-d $\left.{ }_{6}\right) \delta 33.3,39.1,40.1,72.1,79.8,81.2,128.0,130.2$, 130.3, 142.2; IR $\left(v, \mathrm{~cm}^{-1}\right) 2124(\mathrm{C} \equiv \mathrm{C}), 1603,1497$ (Ar), 1360, $1174\left(\mathrm{SO}_{2}-\mathrm{OR}\right)$; MS (GCT, EI): m/z $143\left(\mathrm{M}^{+}-\mathrm{MsO}, 60\right), 91$ (100).

$\mathrm{MsO}$

4-Acetoxybut-2-ynylmethanesulfonate (2g): ${ }^{12}{ }^{1} \mathrm{H}$ NMR (400 MHz, acetone- $\left.\mathrm{d}_{6}\right) \delta 2.05(\mathrm{~s}, 3 \mathrm{H}), 3.19(\mathrm{~s}, 3 \mathrm{H}), 4.78(\mathrm{t}, 2 \mathrm{H}, J=$ $1.8 \mathrm{~Hz}), 4.98(\mathrm{~s}, 2 \mathrm{H}, J=1.8 \mathrm{~Hz}) ;{ }^{13} \mathrm{C}$ NMR $\left(100 \mathrm{MHz}\right.$, acetone-d $\left.{ }_{6}\right) \delta 21.5,39.5,53.2$, 59.4, 80.7, 85.6, 171.3; IR ( $\left.v, \mathrm{~cm}^{-1}\right)$ 1360, 1173 (SO $\left.\mathrm{SO}_{2} \mathrm{OR}\right)$; MS (GCT, EI): $\mathrm{m} / z 111\left(\mathrm{M}^{+}-\right.$ $\mathrm{MsO}, 100 \%)$.

$\mathrm{MsO}$

4-(Benzyloxy)but-2-ynylmethanesulfonate (2h): ${ }^{4}{ }^{1} \mathrm{H}$ NMR $\left(400 \mathrm{MHz}\right.$, acetone- $\left.\mathrm{d}_{6}\right) \delta 3.20(\mathrm{~s}, 3 \mathrm{H}), 4.30(\mathrm{t}, 2 \mathrm{H}, J=1.8 \mathrm{~Hz})$, $4.60(\mathrm{~s}, 2 \mathrm{H}), 5.01(\mathrm{t}, 2 \mathrm{H}, J=1.8 \mathrm{~Hz}), 7.29-7.37(\mathrm{~m}, 5 \mathrm{H}) ;{ }^{13} \mathrm{C}$ NMR $(125 \mathrm{MHz}$, acetone$\left.\mathrm{d}_{6}\right) \delta 39.5,58.7,59.6,73.2,80.9,87.4,129.5,129.7,130.2,139.8$; IR $\left(v, \mathrm{~cm}^{-1}\right) 1604$, 1497 (Ar), 1356, 1175 (SO $\left.\mathrm{SO}_{2} \mathrm{OR}\right)$; MS (GCT, EI): m/z 254 ( $\left.\mathrm{M}^{+}, 1 \%\right), 159$ (M $\left.{ }^{+}-\mathrm{MsO}, 5\right)$, $91(100)$.

$\mathrm{MsO}$

3-Phenylprop-2-ynylmethanesulfonate (2i): ${ }^{13}{ }^{1} \mathrm{H}$ NMR (400 MHz, acetone- $\left.\mathrm{d}_{6}\right) \delta 3.24(\mathrm{~s}, 3 \mathrm{H}), 5.17(\mathrm{~s}, 2 \mathrm{H}), 7.39-7.54(\mathrm{~m}, 5 \mathrm{H}) ;{ }^{13} \mathrm{C} \mathrm{NMR}$ $\left(125 \mathrm{MHz}\right.$, acetone- $\left.\mathrm{d}_{6}\right) \delta 38.1,58.6,82.1,88.6,122.0,129.1,129.8,132.1 ; \mathrm{IR}\left(\mathrm{v}, \mathrm{cm}^{-1}\right)$ 2227 (C $\equiv \mathrm{C}), 1604,1491$ (Ar), 1356, 1174 ( $\left.\mathrm{SO}_{2}-\mathrm{OR}\right)$; MS (GCT, EI): $m / z 210\left(\mathrm{M}^{+}\right)$.

Preparation of allenylmethylsilanes $1 .{ }^{14}$ To an ice-cooled suspension of $\mathrm{CuCN}$ (393 mg, $4.38 \mathrm{mmol})$ and $\mathrm{LiCl}(372 \mathrm{mg}, 8.77 \mathrm{mmol})$ in dry THF $(5.75 \mathrm{~mL})$ was added a solution of $\mathrm{Me}_{3} \mathrm{SiCH}_{2} \mathrm{Mg}(4.38 \mathrm{~mL}, 1.0 \mathrm{M})$ in $\mathrm{Et}_{2} \mathrm{O}$. After being stirred for $25 \mathrm{~min}$, the mixture was cooled to $-78{ }^{\circ} \mathrm{C}$ and a solution of the corresponding mesylate 2 (1.46 $\mathrm{mmol})$ in dry THF $(1.5 \mathrm{~mL})$ was added. After $2 \mathrm{~h}$ at $-78^{\circ} \mathrm{C}$, the reaction was quenched by addition of a saturated aqueous solution of $\mathrm{NH}_{4} \mathrm{Cl}$. The reaction mixture was extracted with $\mathrm{Et}_{2} \mathrm{O}(3 \times 10 \mathrm{~mL})$, washed with brine $(1 \times 15 \mathrm{~mL})$, dried over $\mathrm{MgSO}_{4}$ and concentrated at reduced pressure affording a crude pure by ${ }^{1} \mathrm{H}$ NMR.

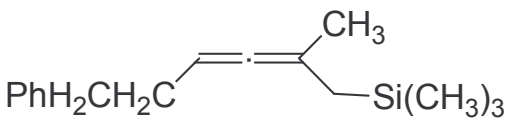

Trimethyl(2-methyl-6-phenylhexa-2,3-dienyl)silane

(1a): ${ }^{1} \mathrm{H}$ NMR $\left(400 \mathrm{MHz}\right.$, acetone- $\left.\mathrm{d}_{6}\right) \delta 0.04(\mathrm{~s}, 9 \mathrm{H})$, $1.29(\mathrm{~s}, 2 \mathrm{H}), 1.60-1.61(\mathrm{~m}, 3 \mathrm{H}), 2.22-2.28(\mathrm{~m}, 2 \mathrm{H})$, $2.70(\mathrm{t}, 2 \mathrm{H}, J=7.6 \mathrm{~Hz}), 4.97-4.98(\mathrm{~m}, 1 \mathrm{H}), 7.14-7.29(\mathrm{~m}, 5 \mathrm{H}) ;{ }^{13} \mathrm{C}$ NMR $(100 \mathrm{MHz}$, acetone- $\left.\mathrm{d}_{6}\right) \delta-0.1,23.2,24.8,33.5,37.3,90.2,98.6,127.5,130.0,130.3,143.9,203.9$; IR $\left(v, \mathrm{~cm}^{-1}\right) 1959(\mathrm{C}=\mathrm{C}=\mathrm{C}), 848(\mathrm{C}-\mathrm{Si})$; HRMS $\left(\mathrm{CI}^{+}\right) \mathrm{m} / z$ calc. for $\mathrm{C}_{16} \mathrm{H}_{25} \mathrm{Si}\left(\mathrm{MH}^{+}\right)$ 245.1726, found 245.1718.<smiles>[B]OCC=C=C(C)C[Si]C</smiles>

[5-(Benzyloxy)-2-methylpenta-2,3dienyl]trimethylsilane (1b): ${ }^{1} \mathrm{H}$ NMR $(400 \mathrm{MHz}$, acetone- $\left.\mathrm{d}_{6}\right) \delta 0.05(\mathrm{~s}, 9 \mathrm{H}), 1.39(\mathrm{~m}, 2 \mathrm{H}), 1.71(\mathrm{~d}, 3 \mathrm{H}, J=$ $2.8 \mathrm{~Hz}), 3.97$ (d, 2H, $J=6.4 \mathrm{~Hz}), 4.51(\mathrm{~s}, 2 \mathrm{H}), 5.08-5.13(\mathrm{~m}, 1 \mathrm{H}), 7.24-7.35(\mathrm{~m}, 5 \mathrm{H})$; ${ }^{13} \mathrm{C}$ NMR $\left(125 \mathrm{MHz}\right.$, acetone- $\left.\mathrm{d}_{6}\right) \delta-1.6,21.5,23.2,69.3,71.4,87.2,98.0,127.6,128.0$,

\footnotetext{
12 Trost, B. M.; Urabe, H. J. Am. Chem. Soc. 1990, 112, 4982-4983.

${ }^{4}$ Wipf, P.; Rahman, L. T.; Rector, S. R. J. Org. Chem. 1998, 63, 7132-7133.

${ }^{13}$ Claesson, A.; Sahlberg, C. Tetrahedron 1982, 38, 363-368.

${ }^{14}$ Nishiyama, T.; Esumi, T.; Iwabuchi, Y.; Irie, H.; Hatakeyama, S. Tetrahedron Lett. 1998, 39, 43-46.
} 
128.5, 139.4, 203.2; IR $\left(v, \mathrm{~cm}^{-1}\right) 1960(\mathrm{C}=\mathrm{C}=\mathrm{C}), 1248,1074(\mathrm{C}-\mathrm{O}), 850(\mathrm{C}-\mathrm{Si})$; HRMS $\left(\mathrm{CI}^{+}\right) \mathrm{m} / z$ calc. for $\mathrm{C}_{16} \mathrm{H}_{28} \mathrm{NOSi}\left(\mathrm{MNH}_{4}^{+}\right)$278.1940, found 278.1936 .<smiles></smiles>

Trimethyl(2-phenyllnona-2,3-dienyl)silane (1c): ${ }^{1} \mathrm{H}$ NMR $\left(400 \mathrm{MHz}\right.$, acetone- $\left.\mathrm{d}_{6}\right) \delta 0.01(\mathrm{~s}, 9 \mathrm{H}), 0.88(\mathrm{t}, 3 \mathrm{H}, J$ $=7.0 \mathrm{~Hz}), 1.30-1.41(\mathrm{~m}, 4 \mathrm{H}), 1.47-1.54(\mathrm{~m}, 2 \mathrm{H}), 1.85(\mathrm{dd}$, $2 \mathrm{H}, J=6.8,2.4 \mathrm{~Hz}), 2.12(\mathrm{q}, 2 \mathrm{H}, J=7.2 \mathrm{~Hz}), 5.44-5.47(\mathrm{~m}, 1 \mathrm{H}), 7.17(\mathrm{t}, 1 \mathrm{H}, J=7.4$ $\mathrm{Hz}), 7.30(\mathrm{t}, 2 \mathrm{H}, J=7.6 \mathrm{~Hz}), 7.43(\mathrm{~d}, 2 \mathrm{H}, J=7.2 \mathrm{~Hz}) ;{ }^{13} \mathrm{C}$ NMR (125 MHz, acetone-d $\left.{ }_{6}\right)$ $\delta-0.6,14.7,20.0,23.5,30.2,30.6,32.6,94.2,104.0,127.4,127.5,129.3,139.7,205.4$; IR $\left(v, \mathrm{~cm}^{-1}\right)$ 1604, $1493(\mathrm{Ar}), 859(\mathrm{C}-\mathrm{Si})$; HRMS $\left(\mathrm{CI}^{+}\right) \mathrm{m} / \mathrm{z}$ calc. for $\mathrm{C}_{18} \mathrm{H}_{29} \mathrm{Si}\left(\mathrm{MH}^{+}\right)$ 273.2039, found 273.2025.<smiles>C[SiH2]CC(C)=C=C[AsH]c1ccccc1</smiles>

Trimethyl(2-methylnona-2,3-dienyl)silane (1d): ${ }^{1} \mathrm{H}$ NMR $\left(400 \mathrm{MHz}, \mathrm{CDCl}_{3}\right) \delta 0.04(\mathrm{~s}, 9 \mathrm{H}), 0.89$ (t, 3H, $J=$ $6.6 \mathrm{~Hz}), 1.26-1.43(\mathrm{~m}, 8 \mathrm{H}), 1.67(\mathrm{~d}, 3 \mathrm{H}, J=2.8 \mathrm{~Hz}), 1.91-$ $1.96(\mathrm{~m}, 2 \mathrm{H}), 4.90-4.93(\mathrm{~m}, 1 \mathrm{H}) ;{ }^{13} \mathrm{C} \mathrm{NMR}\left(125 \mathrm{MHz}, \mathrm{CDCl}_{3}\right) \delta-0.8,14.5,22.5,23.0$, 24.0, 29.5, 30.2, 31.8, 89.6, 96.8, 202.2; IR $\left(v, \mathrm{~cm}^{-1}\right) 849$ (C-Si); HRMS (CI $\left.{ }^{+}\right) \mathrm{m} / z$ calc. for $\mathrm{C}_{13} \mathrm{H}_{27} \mathrm{Si}\left(\mathrm{MH}^{+}\right)$211.1882, found 211.1891.

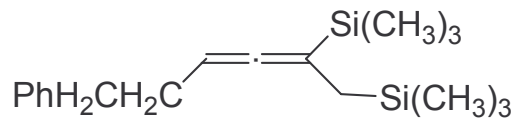

1-[5,6-bis(trimethylsilyl)hexa-3,4-dienyl]benzene (1e): ${ }^{1} \mathrm{H}$ NMR (400 MHz, acetone-d $\left.\mathrm{d}_{6}\right) \delta 0.04(\mathrm{~s}, 9 \mathrm{H})$, $0.07(\mathrm{~s}, 9 \mathrm{H}), 1.29(\mathrm{~d}, 2 \mathrm{H}, J=2.0 \mathrm{~Hz}), 2.25-2.31(\mathrm{~m}$, 2H), $2.71(\mathrm{t}, 2 \mathrm{H}, J=7.8 \mathrm{~Hz}), 4.80-4.84(\mathrm{~m}, 1 \mathrm{H}), 7.15-7.22(\mathrm{~m}, 3 \mathrm{H}), 7.26-7.29(\mathrm{~m}, 2 \mathrm{H})$; ${ }^{13} \mathrm{C}$ NMR (100 MHz, acetone- $\left.\mathrm{d}_{6}\right) \delta-0.3,0.2,18.0,32.9,37.8,86.1,94.3,127.6,130.1$, 130.2, 143.8, 207.4; IR $\left(v, \mathrm{~cm}^{-1}\right) 1927(\mathrm{C}=\mathrm{C}=\mathrm{C}), 1605,1497(\mathrm{Ph}), 840(\mathrm{C}-\mathrm{Si})$; HRMS $\left(\mathrm{CI}^{+}\right) \mathrm{m} / z$ calc. for $\mathrm{C}_{18} \mathrm{H}_{31} \mathrm{Si}_{2}\left(\mathrm{MH}^{+}\right)$303.1964, found 303.1952.<smiles>C[SiH2]CC=C=CCCc1ccccc1</smiles>

Trimethyl(6-phenylhexa-2,3-dienyl)silane (1f): ${ }^{6} \quad{ }^{1} \mathrm{H}$ NMR $\left(400 \mathrm{MHz}\right.$, acetone-d $\left.{ }_{6}\right) \delta 0.02(\mathrm{~s}, 9 \mathrm{H}), 1.26-128$ $(\mathrm{m}, 2 \mathrm{H}), 2.23-2.31(\mathrm{~m}, 2 \mathrm{H}), 2.71(\mathrm{t}, 2 \mathrm{H}, J=7.6 \mathrm{~Hz})$, 5.04-5.12 (m, 2H), 7.15-7.29 (m, 5H); ${ }^{13} \mathrm{C}$ NMR (100 MHz, acetone- $\left.\mathrm{d}_{6}\right) \delta-2.3,17.9$, $31.8,35.9,87.6,89.8,126.1,128.6,128.8,142.3,204.8 ;$ IR $\left(v, \mathrm{~cm}^{-1}\right) 1958(\mathrm{C}=\mathrm{C}=\mathrm{C})$, 1604, 1496 (Ph), 856 (C-Si); MS (GCT, EI): $m / z 230\left(\mathrm{M}^{+}\right)$.

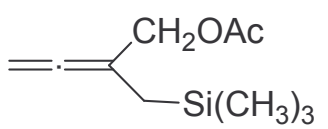

2-[(Trimethylsilyl)methyl]buta-2,3-dienyl acetate $(\mathbf{1 g}):^{12}{ }^{1} \mathrm{H}$ NMR (400 MHz, acetone- $\left.\mathrm{d}_{6}\right) \delta 0.07(\mathrm{~s}, 9 \mathrm{H}), 1.35-1.38(\mathrm{~m}, 2 \mathrm{H})$, $2.01(\mathrm{~s}, 3 \mathrm{H}), 4.45(\mathrm{~m}, 2 \mathrm{H}), 4.76-4.79(\mathrm{~m}, 2 \mathrm{H}) ;{ }^{13} \mathrm{C}$ NMR $(125$ MHz, acetone- $\left.\mathrm{d}_{6}\right) \delta \quad-0.8,18.5,21.2,66.4,77.1,98.5,171.0,208.1 ; \mathrm{IR}\left(\mathrm{v}, \mathrm{cm}^{-1}\right) 1956$ $(\mathrm{C}=\mathrm{C}=\mathrm{C}), 1743(\mathrm{C}=\mathrm{O}), 1249(\mathrm{C}-\mathrm{O}), 849(\mathrm{C}-\mathrm{Si})$; MS $\left(\mathrm{GCT}, \mathrm{CI}^{+}\right): m / z 199\left(\mathrm{MH}^{+}\right)$.

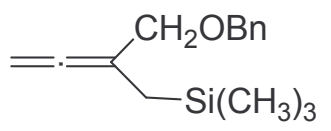

$\{$ 2-[(benzyloxy)methyl]buta-2,3-dienyl $\}$ trimethylsilane $(\mathbf{1 h}):^{14}$ ${ }^{1} \mathrm{H}$ NMR $\left(400 \mathrm{MHz}\right.$, acetone-d $\left.{ }_{6}\right) \delta 0.05(\mathrm{~s}, 9 \mathrm{H}), 1.41(\mathrm{t}, 2 \mathrm{H}, J=2.4$ $\mathrm{Hz}), 3.96(\mathrm{t}, 2 \mathrm{H}, J=2.4 \mathrm{~Hz}), 4.48(\mathrm{~s}, 2 \mathrm{H}), 4.73(\mathrm{~m}, 2 \mathrm{H}), 7.26-7.35$

\footnotetext{
${ }^{6}$ Harada, T.; Katsuhira, T.; Osada, A.; Iwazaki, K.; Maejima, K.; Oku, A. J. Am. Chem. Soc. 1996, 118, 11377-11390.

12 Trost, B. M.; Urabe, H. J. Am. Chem. Soc. 1990, 112, 4982-4983.

${ }^{14}$ Nishiyama, T.; Esumi, T.; Iwabuchi, Y.; Irie, H.; Hatakeyama, S. Tetrahedron Lett. 1998, 39, 43-46.
} 
$(\mathrm{m}, 5 \mathrm{H}) ;{ }^{13} \mathrm{C}$ NMR $\left(125 \mathrm{MHz}\right.$, acetone-d $\left.{ }_{6}\right) \delta-0.6,18.4,72.4,73.3,75.8,99.2,128.6$, 128.9, 129.4, 140.1, 208.6; IR $\left(v, \mathrm{~cm}^{-1}\right) 1954(\mathrm{C}=\mathrm{C}=\mathrm{C}), 1497$ (Ar), 850 (C-Si); MS $\left(\mathrm{GCT}, \mathrm{CI}^{+}\right): m / z 247\left(\mathrm{MH}^{+}\right)$.

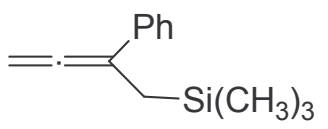

Trimethyl(2-phenylbuta-2,3-dienyl)silane (1i): ${ }^{14}{ }^{1} \mathrm{H}$ NMR (400 MHz, acetone- $\left.\mathrm{d}_{6}\right) \delta 0.004(\mathrm{~s}, 9 \mathrm{H}), 1.85(\mathrm{t}, 2 \mathrm{H}, J=2.4 \mathrm{~Hz}), 5.05(\mathrm{t}$, $2 \mathrm{H}, J=2.4 \mathrm{~Hz}), 7.17-7.45(\mathrm{~m}, 5 \mathrm{H}) ;{ }^{13} \mathrm{C}$ NMR $(125 \mathrm{MHz}$, acetone$\left.\mathrm{d}_{6}\right) \delta-0.6,19.4,77.8,103.5,127.5,127.8,129.4,138.6,210.5 ; \mathrm{IR}\left(v, \mathrm{~cm}^{-1}\right) 1936$ $(\mathrm{C}=\mathrm{C}=\mathrm{C}), 1596,1493(\mathrm{Ar}), 849(\mathrm{C}-\mathrm{Si}) ; \mathrm{MS}\left(\mathrm{GCT}, \mathrm{CI}^{+}\right): m / z 203\left(\mathrm{MH}^{+}\right)$.

Preparation of fluorodienes 4. To a stirred solution of the corresponding allenylmethylsilane $1(0.63 \mathrm{mmol})$ in dry acetone $(6 \mathrm{~mL})$ was added $\mathrm{NaHCO}_{3}(63 \mathrm{mg}$, $0.75 \mathrm{mmol})$ and Selectfluor ${ }^{\mathrm{TM}}(259 \mathrm{mg}, 0.63 \mathrm{mmol})$ under an argon atmosphere. Stirring was continued at room temperature. The solvent was then removed at reduced pressure and hexane $(15 \mathrm{~mL})$ was then added. The suspension was filtered through a pack of neutral alumina and washed with hexane. Solvent was evaporated at reduced pressure $(15 \mathrm{mmHg})$ affording the corresponding fluorodiene.

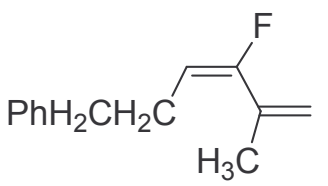

1-[(E)-4-fluoro-5-methylhexa-3,5-dienyl $]$ benzene (4a): The desired product was obtained as a mixture $\mathrm{E} / \mathrm{Z}=2 / 1 ;{ }^{1} \mathrm{H}$ NMR $\left(400 \mathrm{MHz}, \mathrm{CDCl}_{3}\right) \delta 1.88(\mathrm{~s}, 3 \mathrm{H}), 2.48(\mathrm{q}, 2 \mathrm{H}, J=7.6 \mathrm{~Hz}), 2.72(\mathrm{t}$, $2 \mathrm{H}, J=7.6 \mathrm{~Hz}), 5.13(\mathrm{bs}, 1 \mathrm{H}), 5.16(\mathrm{~m}, 1 \mathrm{H}), 5.23(\mathrm{dt}, 1 \mathrm{H}, J=$ 23.6, 8.0 Hz), 7.19-7.33 (m, 5H); ${ }^{13} \mathrm{C}$ NMR (100 MHz, acetone- $\left.\mathrm{d}_{6}\right) \delta 21.2,29.7(\mathrm{~d}, J=$ $8.3 \mathrm{~Hz}), 37.8,109.4$ (d, $J=25.3 \mathrm{~Hz}), 119.3(\mathrm{~d}, J=7.5 \mathrm{~Hz}), 127.8,130.1,130.3,137.4$ $(\mathrm{d}, J=36.3 \mathrm{~Hz}), 143.2,160.2(\mathrm{~d}, J=237.7 \mathrm{~Hz}) ;{ }^{19} \mathrm{~F}$ NMR $\left(376.56 \mathrm{MHz}, \mathrm{CDCl}_{3}\right) \delta-$ 106.0; IR $\left(v, \mathrm{~cm}^{-1}\right) 1672(\mathrm{C}=\mathrm{C}), 1604,1496(\mathrm{Ph})$; HRMS $\left(\mathrm{CI}^{+}\right) \mathrm{m} / z$ calc. for $\mathrm{C}_{13} \mathrm{H}_{19} \mathrm{NF}$ $\left(\mathrm{MNH}_{4}{ }^{+}\right)$208.1502, found 208.1499.

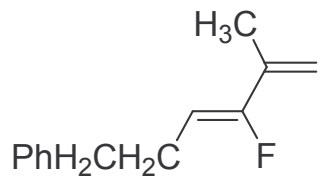

1-[(Z)-4-fluoro-5-methylhexa-3,5-dienyl]benzene (4a'): The desired product was obtained as a mixture $\mathrm{E} / \mathrm{Z}=2 / 1 ;{ }^{1} \mathrm{H} \mathrm{NMR}$ $\left(400 \mathrm{MHz}, \mathrm{CDCl}_{3}\right) \delta 1.88(\mathrm{~s}, 3 \mathrm{H}), 2.53(\mathrm{q}, 2 \mathrm{H}, J=7.6 \mathrm{~Hz}), 2.74(\mathrm{t}$, $2 \mathrm{H}, J=7.6 \mathrm{~Hz}), 4.96(\mathrm{dt}, 1 \mathrm{H}, J=36.4,7.6 \mathrm{~Hz}), 4.99(\mathrm{bs}, 1 \mathrm{H}), 5.34$ (bs, 1H), 7.19-7.33 (m, 5H); ${ }^{13} \mathrm{C}$ NMR (100 MHz, acetone-d 6$) \delta 19.8,27.8(\mathrm{~d}, J=5.0$ $\mathrm{Hz}), 37.0,108.8$ (d, $J=16.8 \mathrm{~Hz}), 113.3$ (d, $J=6.0 \mathrm{~Hz}), 128.0,130.1,130.3,137.0$ (d, $J$ $=29.5 \mathrm{~Hz}), 143.4,159.5(\mathrm{~d}, J=246.9 \mathrm{~Hz}) ;{ }^{19} \mathrm{~F} \mathrm{NMR}\left(376.56 \mathrm{MHz}, \mathrm{CDCl}_{3}\right) \delta-120.8$; IR $\left(v, \mathrm{~cm}^{-1}\right) 1672(\mathrm{C}=\mathrm{C}), 1604,1496(\mathrm{Ph})$; $\mathrm{HRMS}\left(\mathrm{CI}^{+}\right) \mathrm{m} / z$ calc. for $\mathrm{C}_{13} \mathrm{H}_{19} \mathrm{NF}\left(\mathrm{MNH}_{4}{ }^{+}\right)$ 208.1502, found 208.1504.

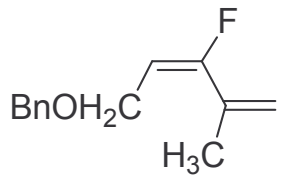

1-\{[(E)-3-Fluoro-4-methylpenta-2,4-dienyloxy $]$ methyl $\}$ benzene (4b): The desired product was obtained as a mixture $\mathrm{E} / \mathrm{Z}=2 / 1 ;{ }^{1} \mathrm{H}$ NMR $\left(400 \mathrm{MHz}, \mathrm{CDCl}_{3}\right) \delta 1.90(\mathrm{~s}, 3 \mathrm{H}), 4.11(\mathrm{dd}, 2 \mathrm{H}, J=7.6,1.2$ $\mathrm{Hz}), 4.51$ (s, 2H), 5.18 (bs, 1H), $5.22(\mathrm{~m}, 1 \mathrm{H}), 5.46$ (dt, 1H, $J=20.8$, $7.6 \mathrm{~Hz}), 7.35-7.37(\mathrm{~m}, 5 \mathrm{H}) ;{ }^{13} \mathrm{C} \mathrm{NMR}\left(100 \mathrm{MHz}, \mathrm{CDCl}_{3}\right) \delta 19.5(\mathrm{~d}, J=1.0 \mathrm{~Hz}), 64.9$ $(\mathrm{d}, J=12.1 \mathrm{~Hz}), 72.1,105.0(\mathrm{~d}, J=25.5 \mathrm{~Hz}), 119.5$ (d, $J=7.9 \mathrm{~Hz}), 127.7,127.8,128.4$, $134.9(\mathrm{~d}, J=28.9 \mathrm{~Hz}), 138.0,161.9(\mathrm{~d}, J=245.8 \mathrm{~Hz}) ;{ }^{19} \mathrm{~F}$ NMR $\left(376.56 \mathrm{MHz}, \mathrm{CDCl}_{3}\right)$ $\delta$-102.0; IR $\left(v, \mathrm{~cm}^{-1}\right) 1672(\mathrm{C}=\mathrm{C}), 1335(\mathrm{C}=\mathrm{CF})$; HRMS $\left(\mathrm{CI}^{+}\right) \mathrm{m} / z$ calc. for $\mathrm{C}_{13} \mathrm{H}_{19} \mathrm{FNO}$ $\left(\mathrm{MNH}_{4}{ }^{+}\right)$224.1451, found 224.1464.

\footnotetext{
${ }^{14}$ Nishiyama, T.; Esumi, T.; Iwabuchi, Y.; Irie, H.; Hatakeyama, S. Tetrahedron Lett. 1998, 39, 43-46.
} 


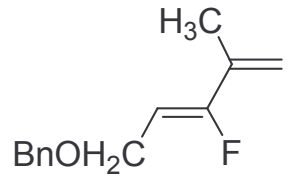
(d, $J=7.0 \mathrm{~Hz}), 72.4,104.0(\mathrm{~d}, J=15.1 \mathrm{~Hz}), 114.2$ (d, $J=6.9 \mathrm{~Hz}), 127.7,127.9,128.4$, $134.4(\mathrm{~d}, J=22.9 \mathrm{~Hz}), 138.1,169.0(\mathrm{~d}, J=251.4 \mathrm{~Hz}) ;{ }^{19} \mathrm{~F}$ NMR $\left(376.56 \mathrm{MHz}, \mathrm{CDCl}_{3}\right)$ $\delta$-117.5; IR $\left(v, \mathrm{~cm}^{-1}\right) 1672(\mathrm{C}=\mathrm{C}), 1335(\mathrm{C}=\mathrm{CF})$; HRMS $\left(\mathrm{CI}^{+}\right) \mathrm{m} / z$ calc. for $\mathrm{C}_{13} \mathrm{H}_{19} \mathrm{FNO}$ $\left(\mathrm{MNH}_{4}^{+}\right)$224.1451, found 224.1448.

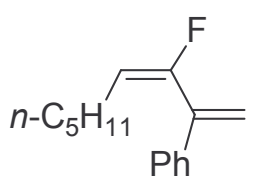

1-[(E)-3-fluoronona-1,3-dien-2-yl]benzene (4c): The desired product was obtained as a mixture $\mathrm{E} / \mathrm{Z}=2 / 1 ;{ }^{1} \mathrm{H}$ NMR $\left(400 \mathrm{MHz}, \mathrm{CDCl}_{3}\right) \delta$ $0.86(\mathrm{t}, 3 \mathrm{H}, J=6.8 \mathrm{~Hz}), 1.20-1.29(\mathrm{~m}, 4 \mathrm{H}), 1.32-1.39(\mathrm{~m}, 2 \mathrm{H}), 1.93(\mathrm{q}$, $2 \mathrm{H}, J=7.6 \mathrm{~Hz}), 5.43(\mathrm{dt}, 1 \mathrm{H}, J=22.0,8.0 \mathrm{~Hz}), 5.48(\mathrm{~m}, 1 \mathrm{H}), 5.69(\mathrm{t}$, $1 \mathrm{H}, J=1.4 \mathrm{~Hz}), 7.32-7.39(\mathrm{~m}, 3 \mathrm{H}), 7.43-7.47(\mathrm{~m}, 2 \mathrm{H}) ;{ }^{13} \mathrm{C} \mathrm{NMR}\left(100 \mathrm{MHz}, \mathrm{CDCl}_{3}\right) \delta$ $14.0,22.4,26.1(\mathrm{~d}, J=7.1 \mathrm{~Hz}), 29.6,31.3,111.1(\mathrm{~d}, J=22.4 \mathrm{~Hz}), 118.6(\mathrm{~d}, J=5.6 \mathrm{~Hz})$, $126.9,128.2,128.4,137.6,140.1(\mathrm{~d}, J=28.5 \mathrm{~Hz}), 156.6(\mathrm{~d}, J=240.3 \mathrm{~Hz}) ;{ }^{19} \mathrm{~F}$ NMR $\left(376.56 \mathrm{MHz}, \mathrm{CDCl}_{3}\right) \delta$-101.6; IR $\left(v, \mathrm{~cm}^{-1}\right) 1686(\mathrm{C}=\mathrm{C}), 1596,1494(\mathrm{Ph})$; HRMS $\left(\mathrm{CI}^{+}\right)$ $m / z$ calc. for $\mathrm{C}_{15} \mathrm{H}_{20} \mathrm{~F}\left(\mathrm{MH}^{+}\right) 219.1549$, found 219.1535 .

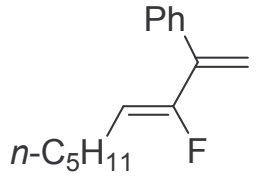

1-[(Z)-3-fluoronona-1,3-dien-2-yl]benzene (4c'): The desired product was obtained as a mixture $\mathrm{E} / \mathrm{Z}=2 / 1 ;{ }^{1} \mathrm{H}$ NMR $(400 \mathrm{MHz}$, $\left.\mathrm{CDCl}_{3}\right) \delta 0.88(\mathrm{t}, 3 \mathrm{H}, J=6.8 \mathrm{~Hz}), 1.22-1.39(\mathrm{~m}, 6 \mathrm{H}), 2.15-2.21(\mathrm{~m}$, $2 \mathrm{H}), 4.81(\mathrm{dt}, 1 \mathrm{H}, J=37.2,7.6 \mathrm{~Hz}), 5.14(\mathrm{~d}, 1 \mathrm{H}, J=2.8 \mathrm{~Hz}), 5.56(\mathrm{~s}$, 1H), 7.36-7.46 (m, 5H); ${ }^{13} \mathrm{C} \mathrm{NMR}\left(100 \mathrm{MHz}, \mathrm{CDCl}_{3}\right) \delta 14.0,22.4,24.3$ (d, $\left.J=5.0 \mathrm{~Hz}\right)$, 28.9, 31.5, 111.7 (d, $J=17.1 \mathrm{~Hz}), 113.6(\mathrm{~d}, J=5.4 \mathrm{~Hz}), 127.8,128.1,128.8,139.1$ (d, $J$ $=5.9 \mathrm{~Hz}), 142.1(\mathrm{~d}, J=26.2 \mathrm{~Hz}), 157.3(\mathrm{~d}, J=246.9 \mathrm{~Hz}) ;{ }^{19} \mathrm{~F}$ NMR $(376.56 \mathrm{MHz}$, $\left.\mathrm{CDCl}_{3}\right) \delta$-120.9; IR $\left(v, \mathrm{~cm}^{-1}\right) 1686(\mathrm{C}=\mathrm{C}), 1596,1494(\mathrm{Ph})$; HRMS $\left(\mathrm{CI}^{+}\right) \mathrm{m} / z$ calc. for $\mathrm{C}_{15} \mathrm{H}_{20} \mathrm{~F}\left(\mathrm{MH}^{+}\right)$219.1549, found 219.1545.

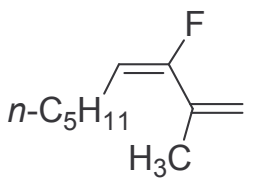

(E)-3-Fluoro-2-methylnona-1,3-diene (4d): The desired product was obtained as a mixture $\mathrm{E} / \mathrm{Z}=2 / 1 ;{ }^{1} \mathrm{H}$ NMR $\left(400 \mathrm{MHz}, \mathrm{CDCl}_{3}\right) \delta 0.89(\mathrm{t}$, $3 \mathrm{H}, J=6.8 \mathrm{~Hz}), 1.26-1.43(\mathrm{~m}, 6 \mathrm{H}), 1.91(\mathrm{~s}, 3 \mathrm{H}), 2.12(\mathrm{q}, 2 \mathrm{H}, J=7.6$ $\mathrm{Hz}), 5.16$ (bs, 2H), 5.17 (dt, $1 \mathrm{H}, J=23.6,8.0 \mathrm{~Hz}) ;{ }^{13} \mathrm{C}$ NMR (100 $\left.\mathrm{MHz}, \mathrm{CDCl}_{3}\right) \delta 13.9,20.1,22.3,25.8(\mathrm{~d}, J=8.25), 29.8,31.3,108.5(\mathrm{~d}, J=23.8 \mathrm{~Hz})$, $117.3(\mathrm{~d}, J=8.8 \mathrm{~Hz}), 135.5(\mathrm{~d}, J=29.8 \mathrm{~Hz}), 157.8(\mathrm{~d}, J=236.4 \mathrm{~Hz}) ;{ }^{19} \mathrm{~F}$ NMR $(376.56$ $\left.\mathrm{MHz}, \mathrm{CDCl}_{3}\right) \delta$-107.6; IR $\left(v, \mathrm{~cm}^{-1}\right) 1672,1615(\mathrm{C}=\mathrm{C})$; HRMS $\left(\mathrm{CI}^{+}\right) \mathrm{m} / z$ calc. for $\mathrm{C}_{10} \mathrm{H}_{21} \mathrm{NF}\left(\mathrm{MNH}_{4}^{+}\right)$174.1658, found 174.1664.

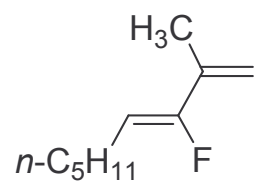

(Z)-3-Fluoro-2-methylnona-1,3-diene (4d'): The desired product was obtained as a mixture $\mathrm{E} / \mathrm{Z}=2 / 1 ;{ }^{1} \mathrm{H}$ NMR $\left(400 \mathrm{MHz}, \mathrm{CDCl}_{3}\right) \delta$ 0.89 (t, 3H, $J=6.8 \mathrm{~Hz}), 1.26-1.43(\mathrm{~m}, 6 \mathrm{H}), 1.84$ (s, 3H), 2.17 (q, 2H, $J$ $=7.6 \mathrm{~Hz}), 4.89(\mathrm{dt}, 1 \mathrm{H}, J=31.6,7.6 \mathrm{~Hz}), 4.94(\mathrm{bs}, 1 \mathrm{H}), 5.29$ (bs, $1 \mathrm{H})$; ${ }^{13} \mathrm{C}$ NMR $\left(100 \mathrm{MHz}, \mathrm{CDCl}_{3}\right) \delta 13.8,18.7(\mathrm{~d}, J=3.9 \mathrm{~Hz}), 22.3,23.9(\mathrm{~d}, J=5.0 \mathrm{~Hz})$, 28.9, 31.3, 107.6 (d, $J=18.8 \mathrm{~Hz}), 111.5(\mathrm{~d}, J=6.3 \mathrm{~Hz}), 134.8(\mathrm{~d}, J=23.6 \mathrm{~Hz}), 157.5$ $(\mathrm{d}, J=245.5 \mathrm{~Hz}) ;{ }^{19} \mathrm{~F}$ NMR $\left(376.56 \mathrm{MHz}, \mathrm{CDCl}_{3}\right) \delta-123.0 ; \mathrm{IR}\left(v, \mathrm{~cm}^{-1}\right) 1672,1615$ $(\mathrm{C}=\mathrm{C})$; HRMS $\left(\mathrm{CI}^{+}\right) \mathrm{m} / z$ calc. for $\mathrm{C}_{10} \mathrm{H}_{21} \mathrm{NF}\left(\mathrm{MNH}_{4}{ }^{+}\right)$174.1658, found 174.1668. 


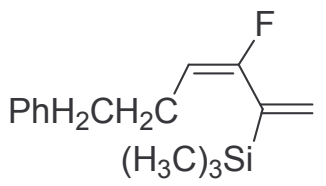

[(E)-3-Fluoro-6-phenylhexa-1,3-dien-2-yl]trimethylsilane (4e): The desired product was obtained as a mixture $\mathrm{E} / \mathrm{Z}=3 / 1 ;{ }^{1} \mathrm{H} \mathrm{NMR}$ $\left(400 \mathrm{MHz}\right.$, acetone- $\left.\mathrm{d}_{6}\right) \delta 0.11(\mathrm{~s}, 9 \mathrm{H}), 2.34(\mathrm{q}, 2 \mathrm{H}, J=7.6 \mathrm{~Hz})$, $2.68(\mathrm{t}, 2 \mathrm{H}, J=7.6 \mathrm{~Hz}), 5.15(\mathrm{dt}, 1 \mathrm{H}, J=22.0,7.6 \mathrm{~Hz}), 5.74-5.75$ $(\mathrm{m}, 1 \mathrm{H}), 5.79(\mathrm{t}, 1 \mathrm{H}, J=3.4 \mathrm{~Hz}), 7.17-7.29(\mathrm{~m}, 5 \mathrm{H}) ;{ }^{13} \mathrm{C}$ NMR $\left(100 \mathrm{MHz}\right.$, acetone-d $\left.\mathrm{d}_{6}\right) \delta$ $-0.5,30.1(\mathrm{~d}, J=7.7 \mathrm{~Hz}), 38.1(\mathrm{~d}, J=1.7 \mathrm{~Hz}), 107.4(\mathrm{~d}, J=25.2 \mathrm{~Hz}), 127.7,130.1$, $130.2,133.0$ (d, $J=11.0 \mathrm{~Hz}), 143.3,145.3(\mathrm{~d}, J=36.4 \mathrm{~Hz}), 163.3(\mathrm{~d}, J=235.0 \mathrm{~Hz}) ;{ }^{19} \mathrm{~F}$ NMR (376.56 MHz, $\left.\mathrm{CDCl}_{3}\right) \delta$-95.5; IR $\left(v, \mathrm{~cm}^{-1}\right) 1673(\mathrm{C}=\mathrm{C}), 1604,1497$ (Ar), 841 (C$\mathrm{Si})$; HRMS $\left(\mathrm{CI}^{+}\right) \mathrm{m} / z$ calc. for $\mathrm{C}_{15} \mathrm{H}_{22} \mathrm{FSi}\left(\mathrm{MH}^{+}\right) 249.1475$, found 249.1467.

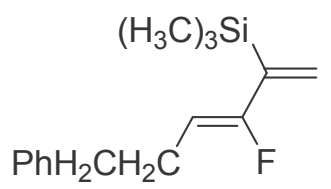

[(Z)-3-Fluoro-6-phenylhexa-1,3-dien-2-yl]trimethylsilane (4e'): The desired product was obtained as a mixture $\mathrm{E} / \mathrm{Z}=3 / 1 ;{ }^{1} \mathrm{H}$ NMR $\left(400 \mathrm{MHz}\right.$, acetone-d $\left.\mathrm{d}_{6}\right) \delta 0.15(\mathrm{~s}, 9 \mathrm{H}), 2.51(\mathrm{~m}, 2 \mathrm{H}), 2.74(\mathrm{t}, 2 \mathrm{H}, J$ $=7.6 \mathrm{~Hz}), 4.98(\mathrm{dt}, 1 \mathrm{H}, J=38.0,7.2 \mathrm{~Hz}), 5.44(\mathrm{~m}, 1 \mathrm{H}), 5.97(\mathrm{~m}$, 1H), 7.17-7.29 (m, 5H); ${ }^{19} \mathrm{~F}$ NMR (376.56 MHz, $\left.\mathrm{CDCl}_{3}\right) \delta$-115.4; IR $\left(v, \mathrm{~cm}^{-1}\right) 1673$ $(\mathrm{C}=\mathrm{C}), 1604,1497(\mathrm{Ar}), 841(\mathrm{C}-\mathrm{Si})$; HRMS $\left(\mathrm{CI}^{+}\right) \mathrm{m} / z$ calc. for $\mathrm{C}_{15} \mathrm{H}_{22} \mathrm{FSi}\left(\mathrm{MH}^{+}\right)$ 249.1475, found 249.1466.

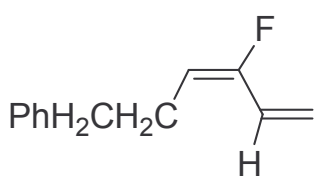

1-[(E)-4-Fluorohexa-3,5-dienyl)benzene (4f): The desired product was obtained as a mixture $\mathrm{E} / \mathrm{Z}=2 / 1 ;{ }^{1} \mathrm{H}$ NMR $(400 \mathrm{MHz}$, acetone- $\left.\mathrm{d}_{6}\right) \delta 2.40(\mathrm{q}, 2 \mathrm{H}, J=7.6 \mathrm{~Hz}), 2.71(\mathrm{t}, 2 \mathrm{H}, J=7.6 \mathrm{~Hz})$, $5.21(\mathrm{~d}, 1 \mathrm{H}, J=10.8 \mathrm{~Hz}), 5.55(\mathrm{~d}, 1 \mathrm{H}, J=17.2 \mathrm{~Hz}), 5.22(\mathrm{dt}, 1 \mathrm{H}, J$ $=19.2,7.6 \mathrm{~Hz}), 6.35(\mathrm{ddd}, 1 \mathrm{H}, J=28.0,17.2,10.8 \mathrm{~Hz}), 7.18-7.32(\mathrm{~m}, 5 \mathrm{H}) ;{ }^{13} \mathrm{C} \mathrm{NMR}$ $\left(100 \mathrm{MHz}\right.$, acetone- $\left.\mathrm{d}_{6}\right) \delta 27.0(\mathrm{~d}, J=8.7 \mathrm{~Hz}), 36.1(\mathrm{~d}, J=2.0 \mathrm{~Hz}), 108.6(\mathrm{~d}, J=22.5$ $\mathrm{Hz}), 115.3(\mathrm{~d}, J=5.3 \mathrm{~Hz}), 124.4(\mathrm{~d}, J=26.3 \mathrm{~Hz}), 128.41,128.43,141.0,156.2(\mathrm{~d}, J=$ $240.7 \mathrm{~Hz}) ;{ }^{19} \mathrm{~F}$ NMR $\left(376.56 \mathrm{MHz}, \mathrm{CDCl}_{3}\right) \delta$-120.4; IR $\left(v, \mathrm{~cm}^{-1}\right) 1673(\mathrm{C}=\mathrm{C}), 1601$, 1496 (Ar); HRMS (EI) $m / z$ calc. for $\mathrm{C}_{12} \mathrm{H}_{13} \mathrm{~F}\left(\mathrm{M}^{+}\right)$176.1001, found 176.0998.

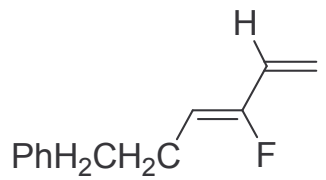

1-[(Z)-4-Fluorohexa-3,5-dienyl)benzene (4f'): The desired product was obtained as a mixture $\mathrm{E} / \mathrm{Z}=2 / 1 ;{ }^{1} \mathrm{H}$ NMR $(400 \mathrm{MHz}$,

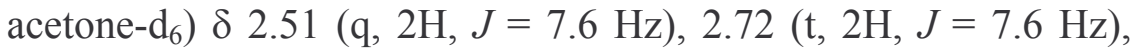
$4.82(\mathrm{dt}, 1 \mathrm{H}, J=36.4,7.6 \mathrm{~Hz}), 5.21(\mathrm{~d}, 1 \mathrm{H}, J=10.8 \mathrm{~Hz}), 5.45(\mathrm{~d}$, $1 \mathrm{H}, J=17.2 \mathrm{~Hz}), 6.08(\mathrm{ddd}, 1 \mathrm{H}, J=26.4,17.2,10.8 \mathrm{~Hz}), 7.18-7.32(\mathrm{~m}, 5 \mathrm{H}) ;{ }^{13} \mathrm{C} \mathrm{NMR}$ $(100 \mathrm{MHz}$, acetone-d 6$) \delta 25.8(\mathrm{~d}, J=4.0 \mathrm{~Hz}), 35.3(\mathrm{~d}, J=1.3 \mathrm{~Hz}), 110.0(\mathrm{~d}, J=16.1$ $\mathrm{Hz}), 113.5$ (d, $J=4.2 \mathrm{~Hz}), 125.9,128.35,128.38,128.9$ (d, $J=26.0 \mathrm{~Hz}), 141.4,156.6$ $(\mathrm{d}, J=247.5 \mathrm{~Hz}) ;{ }^{19} \mathrm{~F}$ NMR $\left(376.56 \mathrm{MHz}, \mathrm{CDCl}_{3}\right) \delta-125.8$; IR $\left(v, \mathrm{~cm}^{-1}\right) 1673(\mathrm{C}=\mathrm{C})$, 1601, 1496 (Ar); HRMS (EI) $m / z$ calc. for $\mathrm{C}_{12} \mathrm{H}_{13} \mathrm{~F}\left(\mathrm{M}^{+}\right)$176.1001, found 176.0992.

$\mathrm{AcOH}_{2} \mathrm{C}$ 3-Fluoro-2-methylenebut-3-enyl acetate (4g): ${ }^{1} \mathrm{H}$ NMR (400 MHz, $\left.\mathrm{CDCl}_{3}\right) \delta 2.10(\mathrm{~s}, 3 \mathrm{H}), 4.61(\mathrm{dd}, 1 \mathrm{H}, J=50.0,3.6 \mathrm{~Hz}), 4.71(\mathrm{~s}, 2 \mathrm{H}), 4.83$ (ddd, $1 \mathrm{H}, J=18.4,3.6,1.2 \mathrm{~Hz}), 5.42(\mathrm{~m}, 1 \mathrm{H}), 5.67$ (bs, $1 \mathrm{H}) ;{ }^{13} \mathrm{C} \mathrm{NMR}$ $\left(125 \mathrm{MHz}, \mathrm{CDCl}_{3}\right) \delta 20.7,63.0(\mathrm{~d}, J=6.3 \mathrm{~Hz}), 91.8(\mathrm{~d}, J=21.3 \mathrm{~Hz})$, $117.2(\mathrm{~d}, J=5.0 \mathrm{~Hz}), 134.1(\mathrm{~d}, J=26.3 \mathrm{~Hz}), 160.5(\mathrm{~d}, J=250.0 \mathrm{~Hz}), 170.4 ;{ }^{19} \mathrm{~F} \mathrm{NMR}$ $\left(376.56 \mathrm{MHz}, \mathrm{CDCl}_{3}\right) \delta$-109.0; IR $\left(v, \mathrm{~cm}^{-1}\right) 1746(\mathrm{C}=\mathrm{O}), 1655,1610(\mathrm{C}=\mathrm{C}), 1338$ $(\mathrm{C}=\mathrm{CF})$; HRMS $\left(\mathrm{CI}^{+}\right) \mathrm{m} / z$ calc. for $\mathrm{C}_{7} \mathrm{H}_{13} \mathrm{NO}_{2} \mathrm{~F}\left(\mathrm{MNH}_{4}^{+}\right)$162.0930, found 162.0928.

$\mathrm{BnOH}_{2} \mathrm{C}$

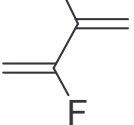

1-[(3-Fluoro-2-methylenebut-3-enyloxy)methyl]benzene (4h): ${ }^{1} \mathrm{H}$

NMR $\left(400 \mathrm{MHz}, \mathrm{CDCl}_{3}\right) \delta 4.16(\mathrm{~s}, 2 \mathrm{H}), 4.53(\mathrm{~s}, 2 \mathrm{H}), 4.71(\mathrm{dd}, 1 \mathrm{H}, J=$ $50.0,3.2 \mathrm{~Hz}), 4.76-4.81(\mathrm{~m}, 1 \mathrm{H}), 5.40(\mathrm{bs}, 1 \mathrm{H}), 5.65(\mathrm{~s}, 1 \mathrm{H}), 7.29-7.40$ 
$(\mathrm{m}, 5 \mathrm{H}) ;{ }^{13} \mathrm{C}$ NMR $\left(100 \mathrm{MHz}, \mathrm{CDCl}_{3}\right) \delta 69.5(\mathrm{~d}, J=5.4 \mathrm{~Hz}), 71.9,91.9(\mathrm{~d}, J=20.8$ $\mathrm{Hz}), 116.3$ (d, $J=5.8 \mathrm{~Hz}), 127.70,127.74,128.4,135.8$ (d, $J=23.6 \mathrm{~Hz}), 137.8,161.1$ $(\mathrm{d}, J=250.1 \mathrm{~Hz}) ;{ }^{19} \mathrm{~F}$ NMR $\left(376.56 \mathrm{MHz}, \mathrm{CDCl}_{3}\right) \delta-108.6$; IR $\left(v, \mathrm{~cm}^{-1}\right) 1654(\mathrm{C}=\mathrm{C})$, 1605, 1497 (Ar), $1336(\mathrm{C}=\mathrm{CF}), 1094(\mathrm{C}-\mathrm{O})$; HRMS $\left(\mathrm{CI}^{+}\right) \mathrm{m} / z$ calc. for $\mathrm{C}_{12} \mathrm{H}_{17} \mathrm{NOF}$ $\left(\mathrm{MNH}_{4}{ }^{+}\right)$210.1294, found 210.1286.

$\mathrm{Ph}$ 1-(3-Fluorobuta-1,3-dien-2-yl)benzene (4i): ${ }^{1} \mathrm{H} \mathrm{NMR}\left(400 \mathrm{MHz}, \mathrm{CDCl}_{3}\right) \delta$

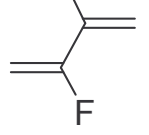
4.49 (dd, 1H, $J=49.4,3.0 \mathrm{~Hz}$ ), 4.86 (ddd, 1H, $J=17.2,3.0,2.4 \mathrm{~Hz}), 5.29$ (m, $1 \mathrm{H}), 5.69(\mathrm{bs}, 1 \mathrm{H}), 7.32-7.37(\mathrm{~m}, 5 \mathrm{H}) ;{ }^{13} \mathrm{C} \mathrm{NMR}\left(100 \mathrm{MHz}, \mathrm{CDCl}_{3}\right) \delta 94.6$ $(\mathrm{d}, J=21.6 \mathrm{~Hz}), 116.1(\mathrm{~d}, J=5.4 \mathrm{~Hz}), 128.0,128.2,128.6,138.6(\mathrm{~d}, J=5.7$ $\mathrm{Hz}), 141.6(\mathrm{~d}, J=26.0 \mathrm{~Hz}), 163.1(\mathrm{~d}, J=251.5 \mathrm{~Hz}) ;{ }^{19} \mathrm{~F} \mathrm{NMR}\left(376.56 \mathrm{MHz}, \mathrm{CDCl}_{3}\right) \delta$ 107.6; IR ( $\left.v, \mathrm{~cm}^{-1}\right) 1643(\mathrm{C}=\mathrm{C}), 1594,1493(\mathrm{Ar}), 1342(\mathrm{C}=\mathrm{CF})$; HRMS $\left(\mathrm{CI}^{+}\right) \mathrm{m} / z$ calc. for $\mathrm{C}_{10} \mathrm{H}_{10} \mathrm{~F}\left(\mathrm{MH}^{+}\right)$149.0767, found 139.0769. 


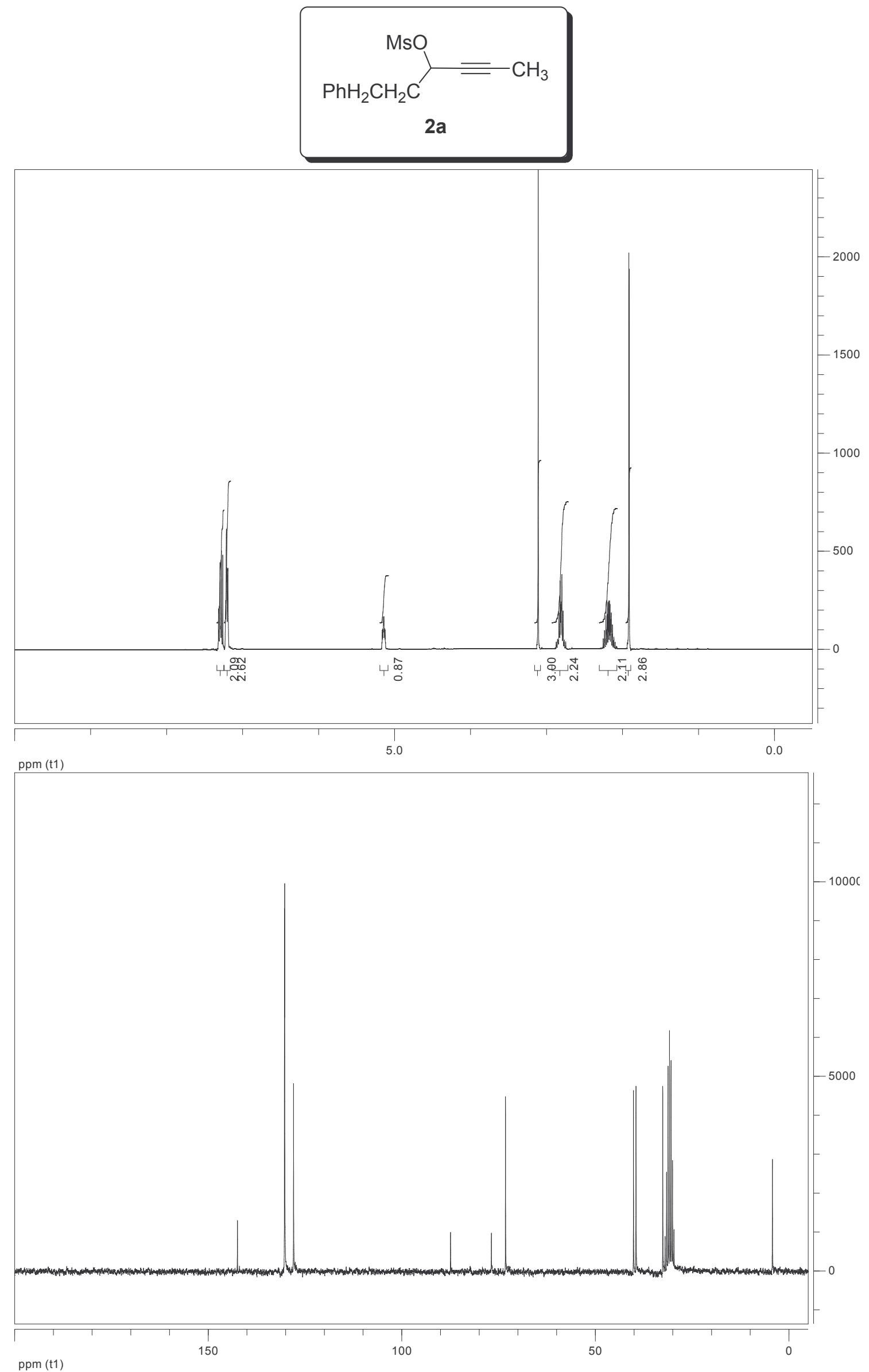




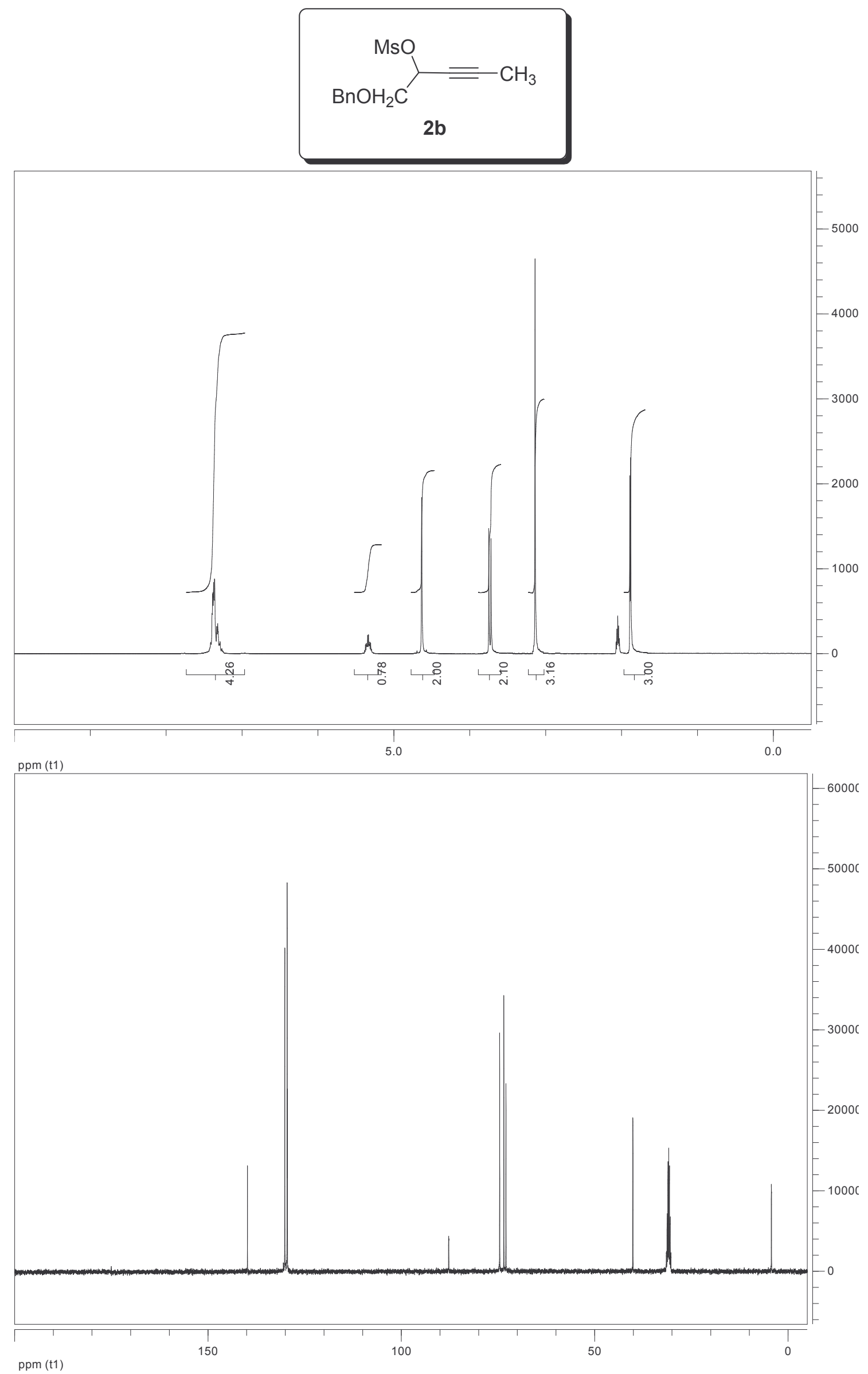




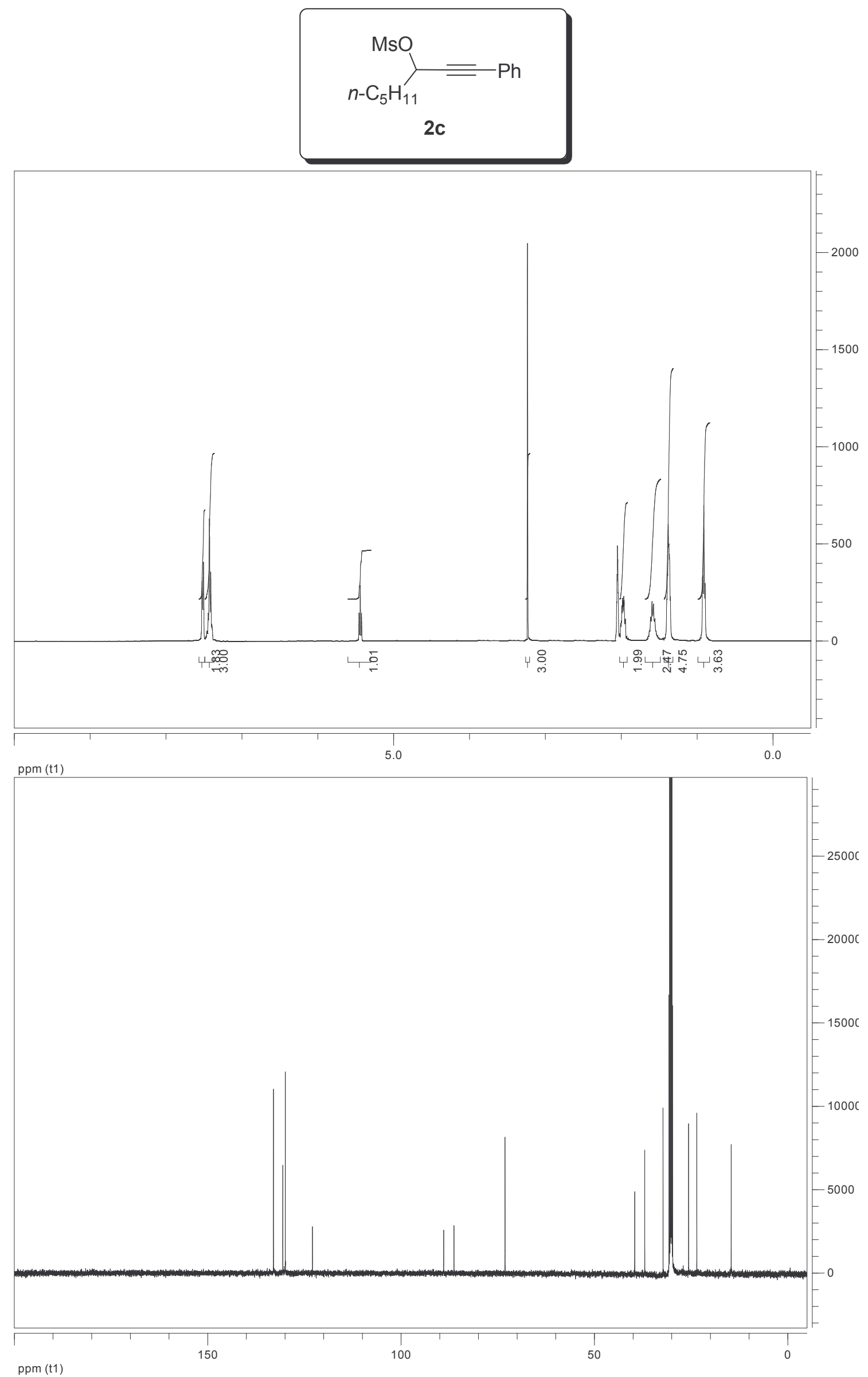




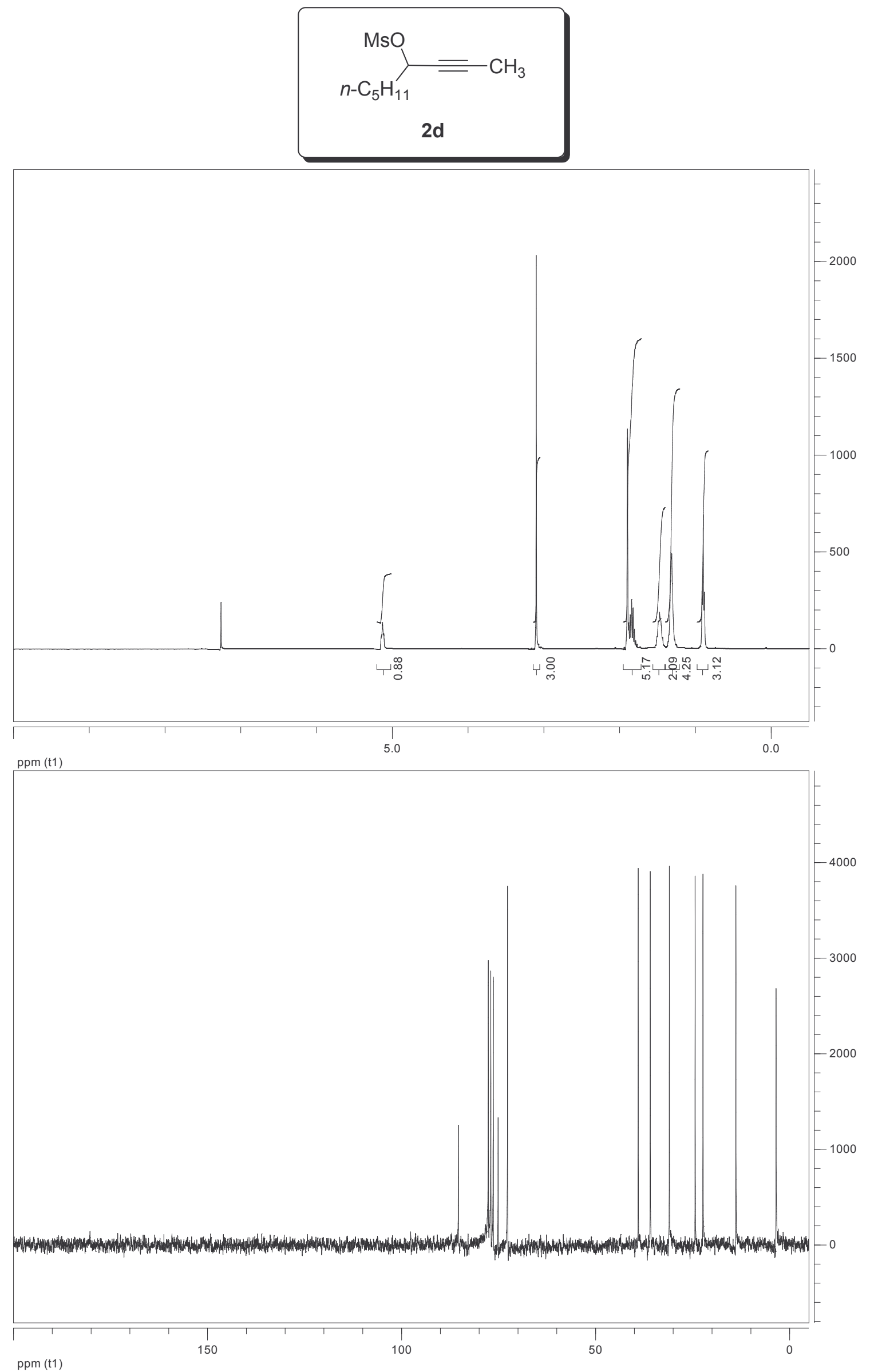




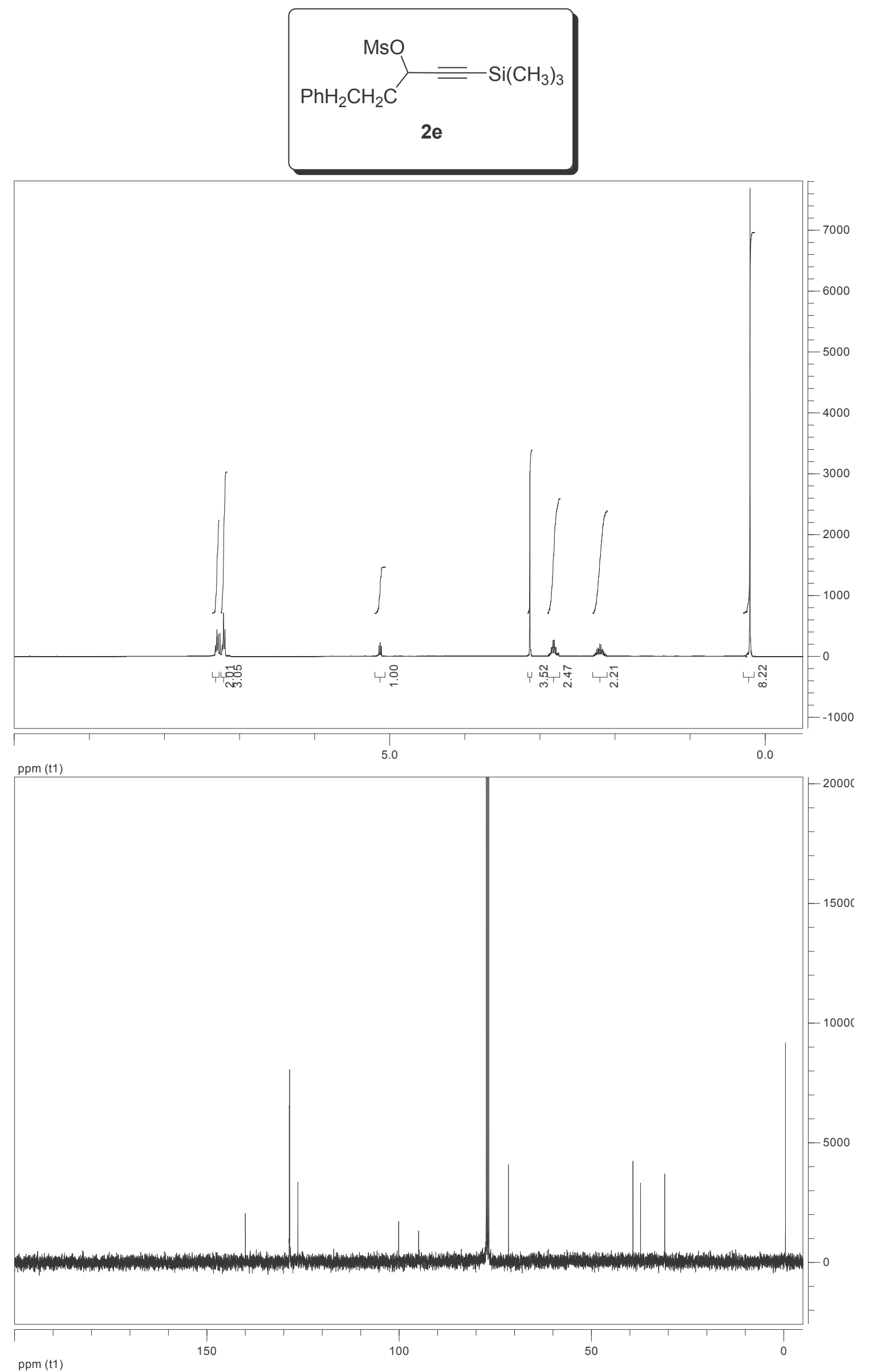




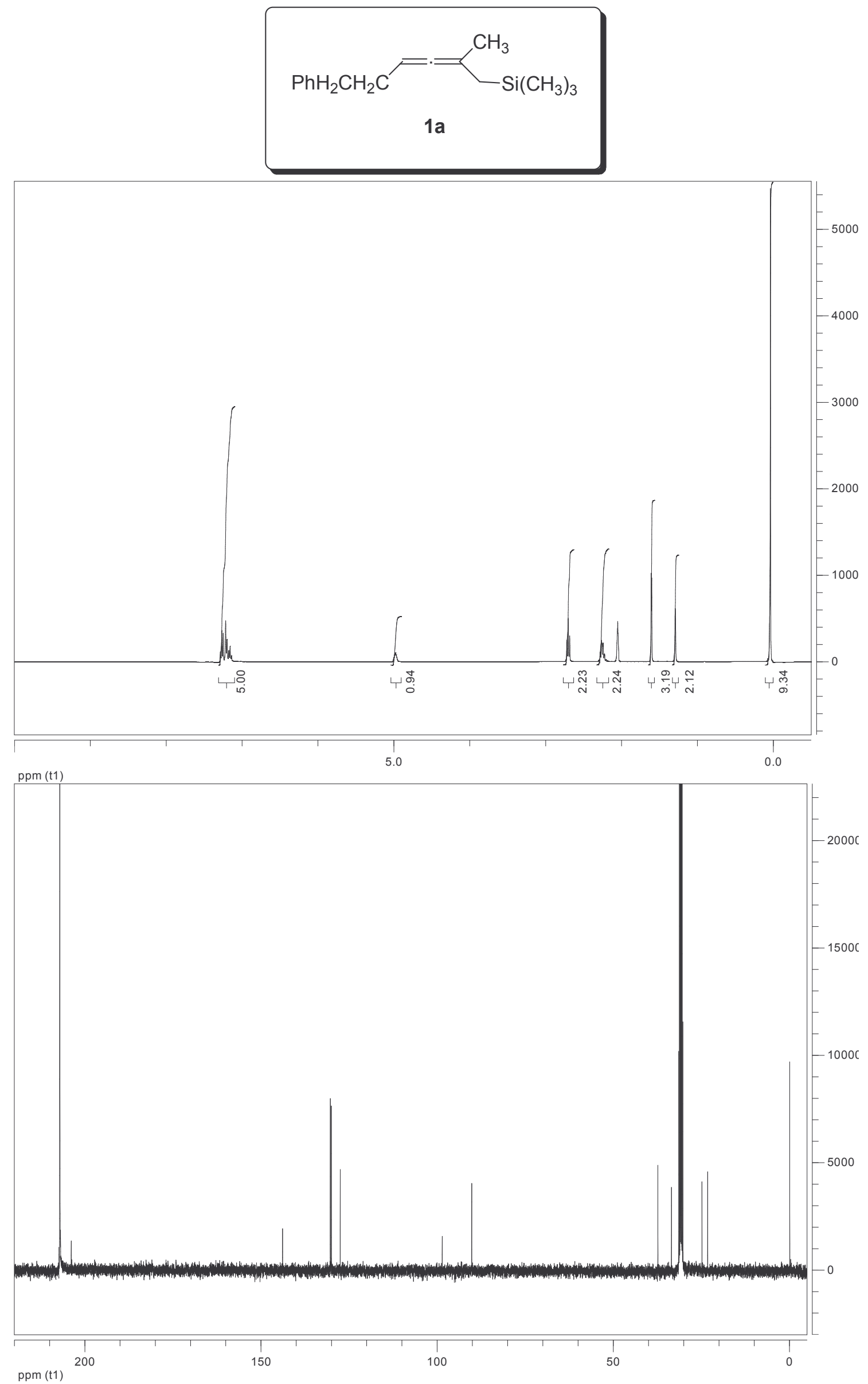




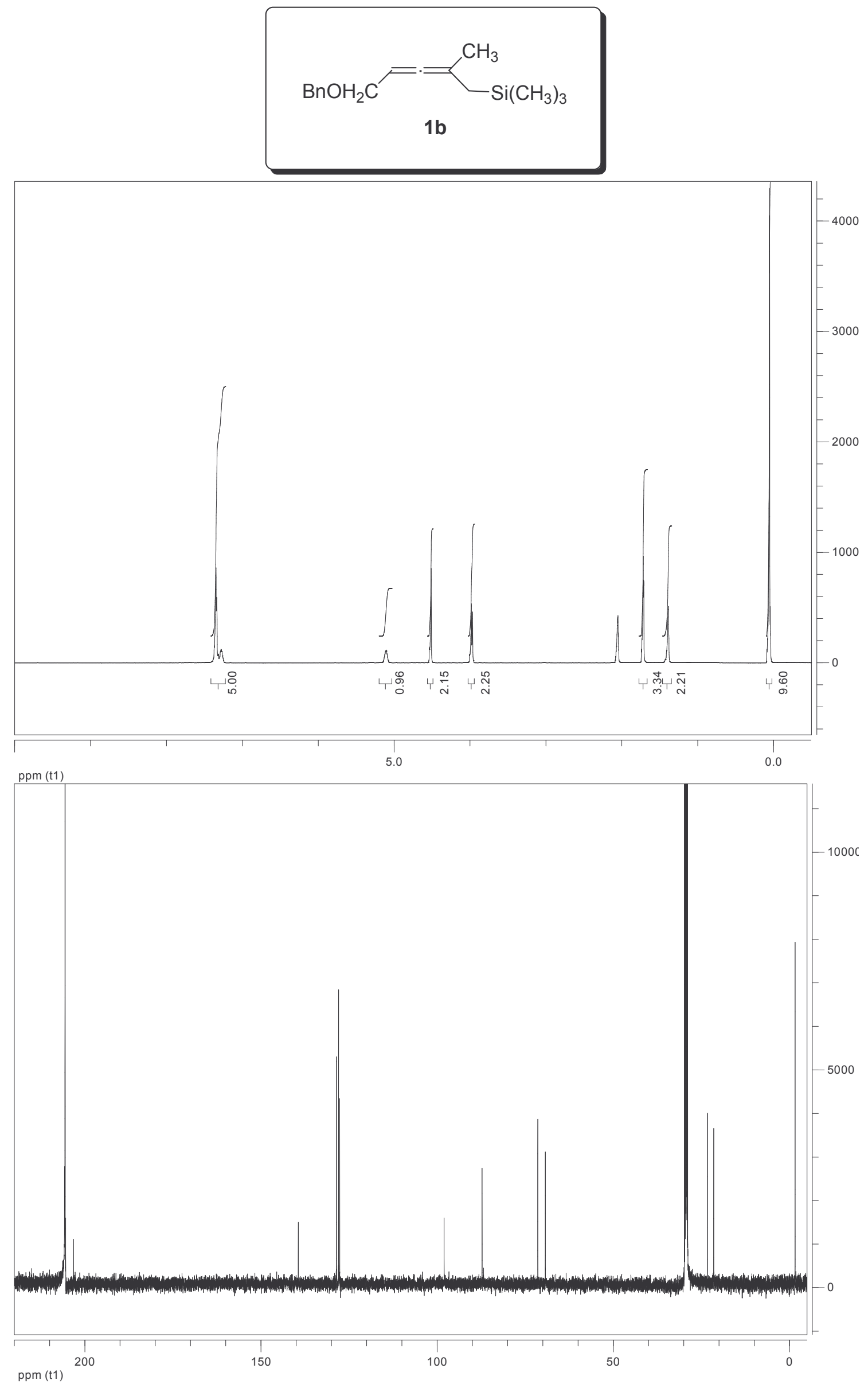




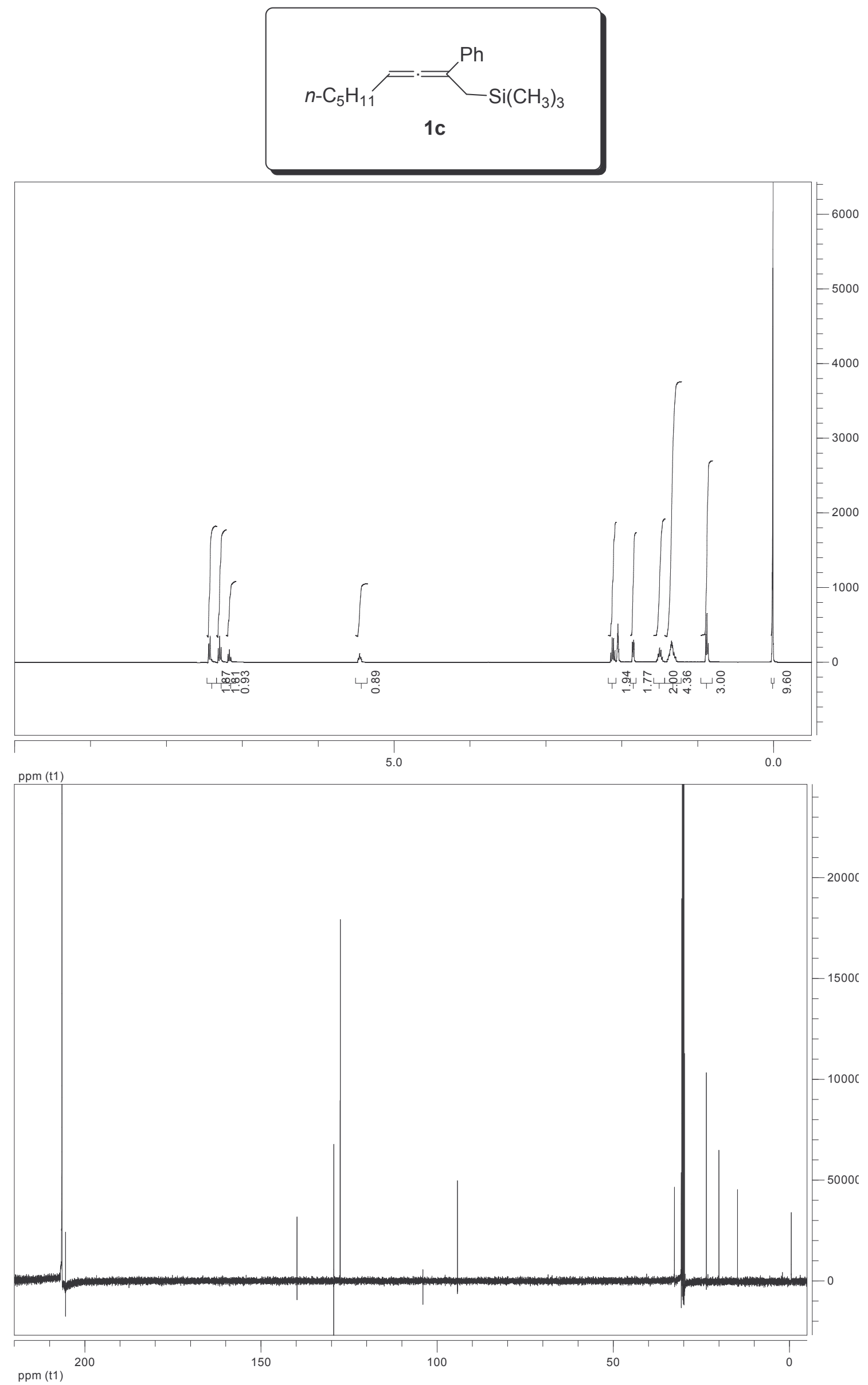




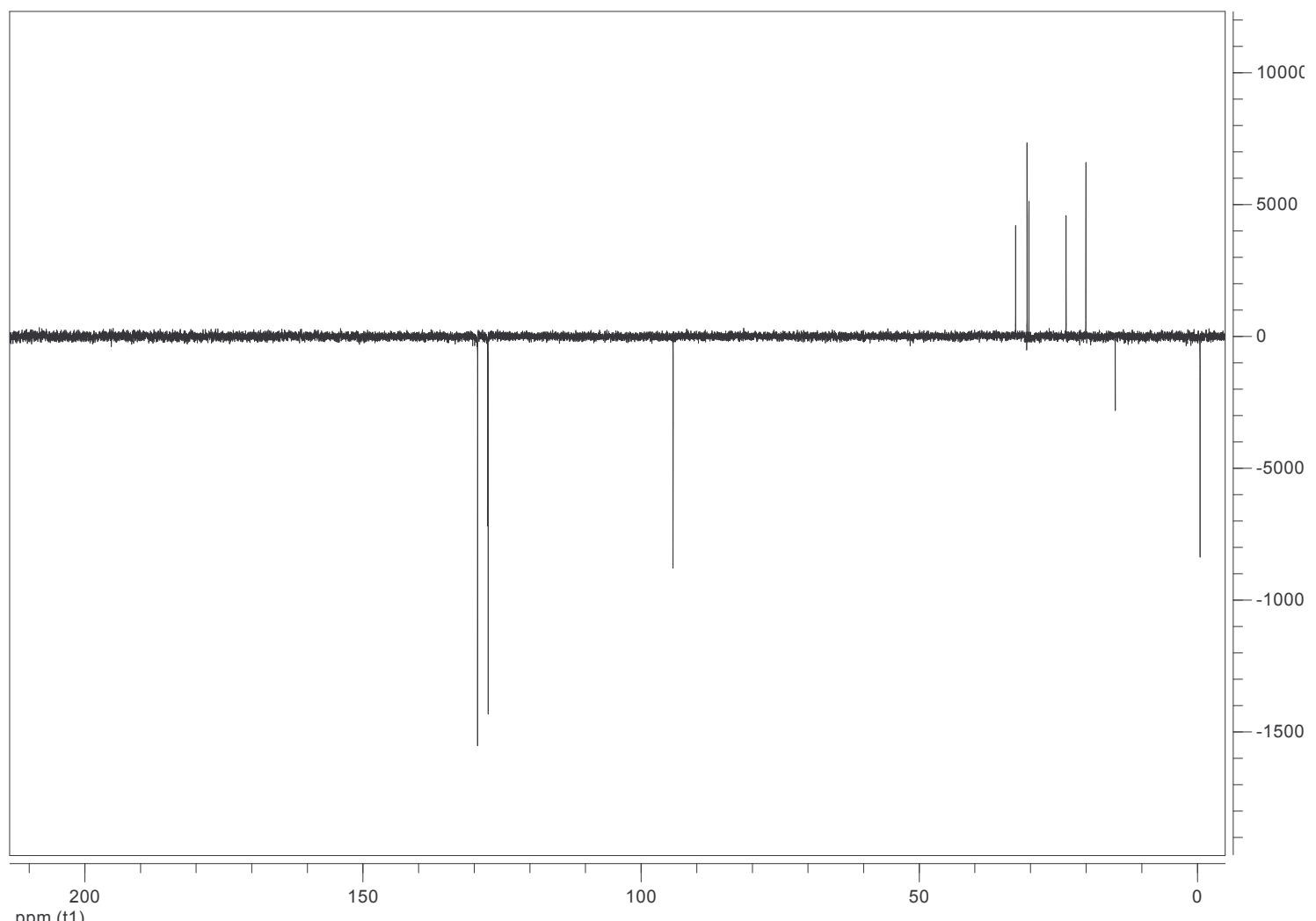

ppm (t1) 


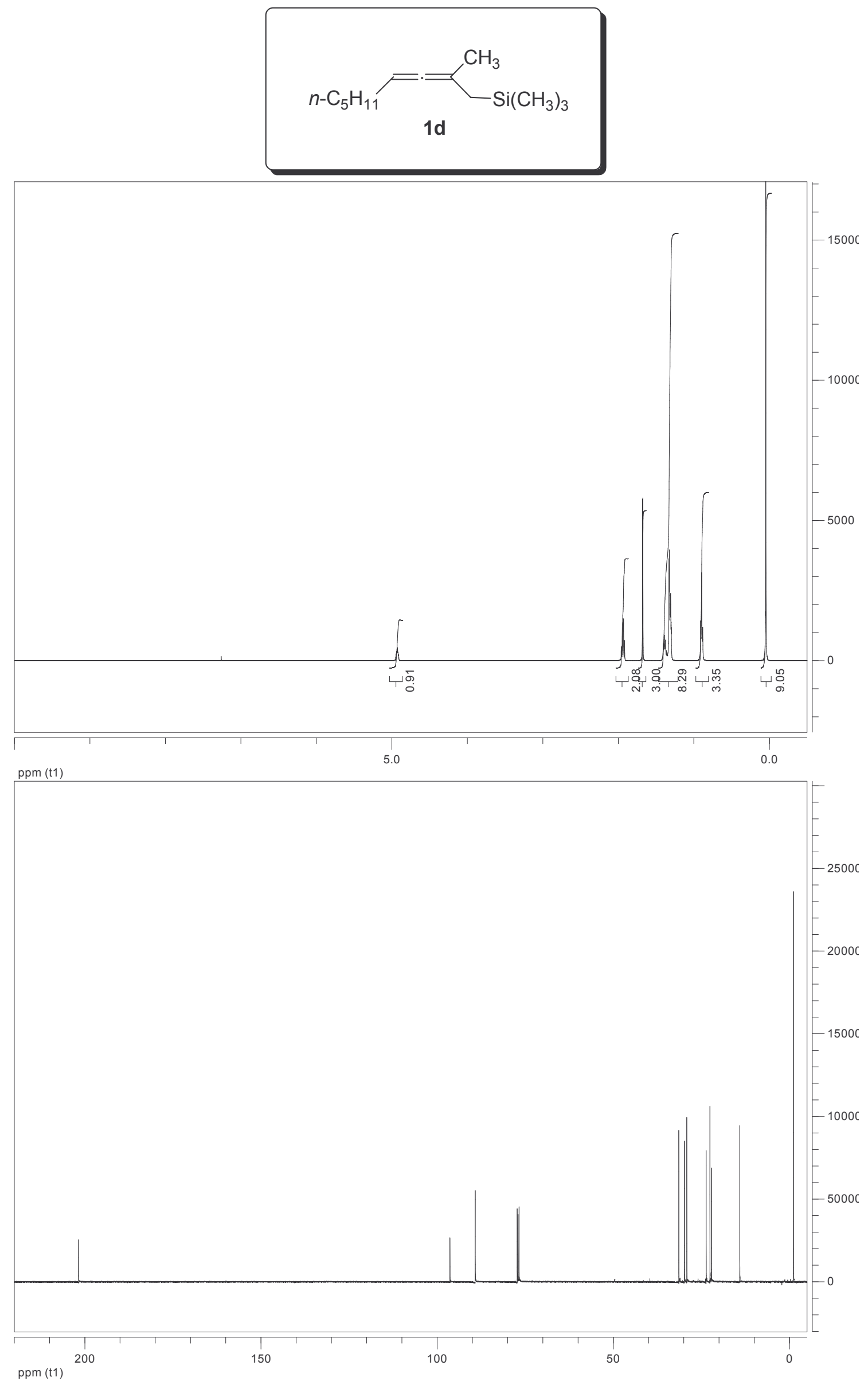




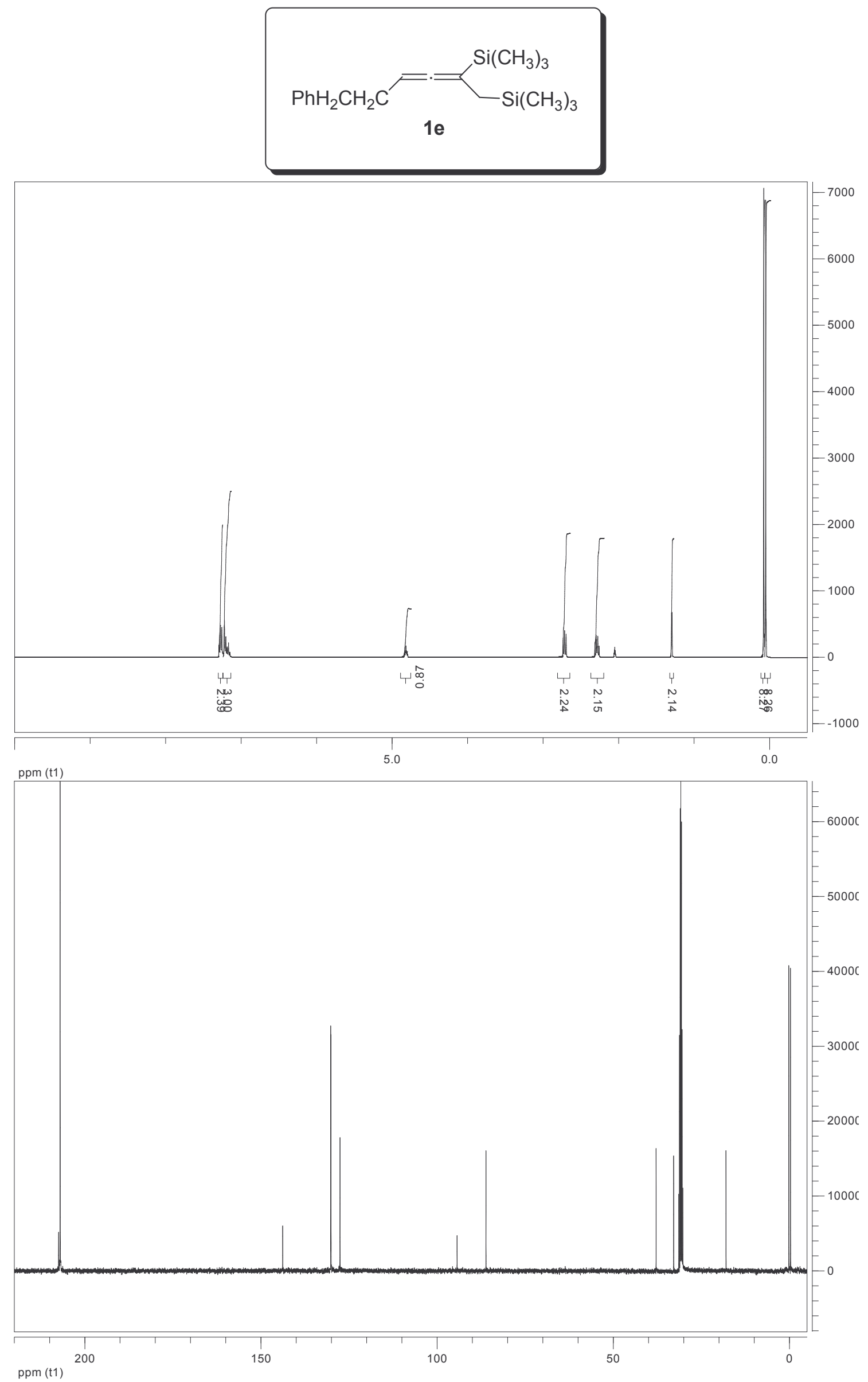

